\title{
Studies on the structure, optical, ac conduction mechanism by OLPT model and impedance spectroscopy of La-doped Ba2SnO4 Ruddlesden Popper Oxide
}

Upendra Kumar ( $\square$ upendra.bhu512@gmail.com )

Indian Institute of Information Technology Allahabad https://orcid.org/0000-0001-9200-0048

Ram Sundar Maurya

Banasthali Vidyapith: Banasthali University

Harshpreet Cheema

Banasthali Vidyapith: Banasthali University

Vedika Yadav

Banasthali Vidyapith: Banasthali University

Aditya Kumar

University of Allahabad

P. A. Alvi

Banasthali Vidyapith: Banasthali University

\section{Research Article}

Keywords: La-doped Ba2SnO4, Rietveld Refinement, Raman spectroscopy; OLPT model, Impedance Spectroscopy.

Posted Date: June 24th, 2021

DOl: https://doi.org/10.21203/rs.3.rs-646048/v1

License: (c) (1) This work is licensed under a Creative Commons Attribution 4.0 International License. Read Full License 


\section{Studies on the structure, optical, ac conduction mechanism by OLPT model and impedance spectroscopy of La-doped $\mathrm{Ba}_{2} \mathrm{SnO}_{4}$ Ruddlesden Popper Oxide}

Upendra Kumar ${ }^{*}$, Ram Sundar Maurya ${ }^{2}$, Harshpreet Cheema², Vedika Yadav², Aditya $\mathrm{Kumar}^{3}$, P. A. Alvi ${ }^{2}$

${ }^{1}$ Department of Applied Science, Indian Institute of Information Technology Allahabad, Jhalwa, Pryagraj-211015, Uttar Pradesh, India

${ }^{2}$ Department of Physics, Banasthali Vidyapith, Banasthali-304022, Rajasthan, India

${ }^{3}$ Center of Material Sciences, University of Allahabad, Prayagraj-211002, India

\section{Abstract}

The conventional ceramic route has been used to prepare the La-doped $\mathrm{Ba}_{2} \mathrm{SnO}_{4}$ samples by heat-treatment at $1000^{\circ} \mathrm{C}$ and sintered at $1250^{\circ} \mathrm{C}$. The phase identification was carried out using $\mathrm{XRD}$ and found to be single phase up to 4 atoms \%. The solubility of La at Ba-site were further reconfirmed using FTIR and Raman analysis. The AC conductivity spectra of all samples follow universal Johnscher's power law $\sigma_{a c}=\sigma_{d c}\left(1+\left(\frac{f}{f_{h}}\right)^{n}\right)$; however, the thermal dependence of $\sigma_{d c}$ and $f_{h}$ suggest Arrhenius type conduction within the sample. Further, the scaled conductivity $\sigma_{a c} / \sigma_{d c}$ and frequency $f / f_{h}$ at all temperatures superimposes on a single master curve, indicates the invariance of conduction mechanism. The impedance spectroscopy studies suggest the major contribution of grain in the conduction and relaxation. The present material could be potentially used in semiconductor device, UV-detector, and mixed ionic and electronic conductor (MIECs) by utilizing absorption states and thermo-curves as metastable state.

Keywords: La-doped $\mathrm{Ba}_{2} \mathrm{SnO}_{4}$, Rietveld Refinement, Raman spectroscopy; OLPT model, Impedance Spectroscopy.

*Corresponding Author

Upendra Kumar (upendra.bhu512@gmail.com) 


\section{Introduction:}

The ternary oxides belonging to the Ruddlesden Popper (RP) phase were identified by the general chemical formula $\mathrm{A}_{n+1} \mathrm{~B}_{\mathrm{n}} \mathrm{O}_{3 n+1}$, consisting of n-layered perovskite $\left(\mathrm{ABO}_{3}\right)$ slabs sandwiched along the c-axis by two rock salt layers of A-O [1-3]. Because of their exceptional electronic and ionic transmission behaviour, this form structure gained considerable interest from researchers in discovering its potential used in superconductors, supercapacitors, as thermoelectric materials, as multiferroic materials and as cathode materials for solid oxide fuel cells [4-9]. Due to their lower thermal expansion coefficients (TECs) than the conventional perovskite-based manganite, the rare earth-based nickelate, cobaltite, and ferrite were more extensively studied among the investigated $\mathrm{K}_{2} \mathrm{NiF}_{4}$ type phase [10-12]. Another advantage of these structures over perovskites is that perovskites have a compact structure, so they only have an oxygen deficiency phase, while RP phases have both an oxygen deficiency and in efficient phase. Because of this exceptional property, these typical structures can be used in mixed ionic and electronic conductor (MIEC) applications [1,13,14].

Rare-earth based nickelate/cobaltite $\mathrm{Ln}_{2} \mathrm{NiO}_{4} / \mathrm{Ln}_{2} \mathrm{CoO}_{4}$ (Ln: $\mathrm{La}, \mathrm{Pr}, \mathrm{Nd}$ ) have been recognized as auspicious cathode materials due to their ability to replace conventional electrode materials used at high temperatures $[7,10,11]$. There are two types of oxygen sites in the structure: an equatorial oxygen site inside the perovskite, and an axial oxygen site that serves as a connection between the perovskite and the rock-salt layer [15]. The interstitial incorporation of oxygen between the perovskite and rock salt layers causes an extraordinarily large change in oxygen non-stoichiometry $(\delta)$ for solid oxide fuel cells at intermediate temperatures (IT-SOFCs). The transition metal was used as a source of conduction in these materials, which was accomplished through an oxidation $\left(\mathrm{Ni}^{2+} / \mathrm{Co}^{2+}\right.$ to $\left.\mathrm{Ni}^{3+} \mathrm{Co}^{3+}\right)$ and reduction $\left(\mathrm{Ni}^{3+} / \mathrm{Co}^{3+}\right.$ to $\left.\mathrm{Ni}^{2+} / \mathrm{Co}^{2+}\right)$ process. A.P. Khandelwal et al. studied the electrical properties of $\mathrm{Nd}_{2} \mathrm{NiO}_{4}$ and reported that the replacement of alkali earth metals at the $\mathrm{Nd}$-site improved mixed electronic and ionic conductivity [16]. By tuning the valence states of $\mathrm{Fe}$, Chao Jin et al. mentioned that $\mathrm{Sr}$ substitution at the Ba-site of $\mathrm{Ba}_{2} \mathrm{FeO}_{4}$ could be used as a new cathode material for IT-SOFCs [17]. Since the chemical used in nickelates is so expansive, it's unlikely that these conventional materials will ever be commercialized in bulk. It is therefore essential to find an alternative to this material, not just for the development of new materials but also for the understanding of the fundamental solid-state physics. It has been noted on the basis of literature that alkaline earth-based stannates $\left(\mathrm{M}_{2} \mathrm{SnO}_{4}, \mathrm{M}\right.$ : $\left.\mathrm{Ca}, \mathrm{Sr}, \mathrm{Ba}\right)$ completely fulfil the requisite requirements 
possessed by $\mathrm{La}_{2} \mathrm{NiO}_{4}, \mathrm{La}_{2} \mathrm{CoO}_{4}, \mathrm{Ba}_{2} \mathrm{FeO}_{4}$, etc. such as, (i) it has multivalent ion $\mathrm{Sn}$, that exist in $\mathrm{Sn}^{4+} / \mathrm{Sn}^{2+}$, (ii) larger the volume for tuning the oxygen non-stoichiometry $(\delta)$.

Alkaline earth based stannates $\left(\mathrm{M}_{2} \mathrm{SnO}_{4}\right)$ also belongs to the RP phase, which belongs to the space group $I 4 / \mathrm{mmm}$ and crystallized into tetragonal structure $(a=b \neq c, \alpha=\beta=\gamma=$ $90^{\circ}$ ) [18]. Alkaline earth orthostannates have recently received more attention as a new phosphor material, owing to their stable crystalline nature and high physical and chemical stability [19]. Rare earth metals and transition metals were used to control the photoluminescence properties of the host phosphor material that are used in flat panel displays, field emission displays, and electroluminescence devices [20,21]. Detailed electrical properties of $\mathrm{Ba}_{2} \mathrm{SnO}_{4}$ have already been described in the literature; two types of conduction mechanisms have been found to be operative within the investigated temperature range, assigned to electronic conduction at low temperatures and ionic conduction at higher temperatures [15]. The modification in $\mathrm{Ba}_{2} \mathrm{SnO}_{4}$ lattice manipulates the sources of charge carriers and semiconducting properties that played the crucial role in the electrical properties. The doping of various atoms in the solids can be done on the basis of Hume-Rothery criteria [22]; the difference of ionic radii between the host and dopants should be lye below $\pm 15 \%$. Since the difference between the ionic radii of La and $\mathrm{Ba}$ was found to be less than $15 \%$, this means that $\mathrm{La}$ is a suitable dopant for the $\mathrm{Ba}$ site of $\mathrm{Ba}_{2} \mathrm{SnO}_{4}$. Since $\mathrm{Ba}_{2} \mathrm{SnO}_{4}$ consists of a perovskite $\mathrm{BaSnO}_{3}$ and rock salt layer $\mathrm{BaO}$, so it will be expecting that it might be show a cumulative property of $\mathrm{BaSnO}_{3}$ and $\mathrm{BaO}$. There are several reports available in literature on La-doped $\mathrm{BaSnO}_{3}$ system [23-30]. Among the investigated papers, following are the investigations; $\mathrm{K}$. K. James et al. prepared the thin film of La-doped $\mathrm{BaSnO}_{3}$ at fused silica substrate using laser ablation, and found a progressive increase in conductivity up to $9 \mathrm{Scm}^{-1}$ with incorporation of 7 at \% La at Ba-site[27]. Q. Liu et al. also prepared the same thin film of $\mathrm{La}$-doped $\mathrm{BaSnO}_{3}$ using laser ablation and found a compositional dependence metal to semiconductor transition below room temperature[28]. Upadhyay et al. synthesized the nanopowder of La-doped $\mathrm{BaSnO}_{3}$ system and studied their electrical properties using impedance spectroscopy and found their application as oxygen ion and hydroxyl ion conductor [29]. Masahiro et al. synthesized the ceramics of La-doped $\mathrm{BaSnO}_{3}$ using polymerized complex method and reported it as ntype degenerate semiconductor [23]. The multifunctional application of La-doped $\mathrm{BaSnO}_{3}$ enforce us to choose La as dopant for present system and will be expect to be as interesting as reported in literature. It is very interesting that no research has been available in the literature 
so far on the partial substitution of $\mathrm{La}$ at the $\mathrm{Ba}$ site in $\mathrm{Ba}_{2} \mathrm{SnO}_{4}$. Therefore, an attempt has been made in this manuscript to replace $\mathrm{Ba}$ with $\mathrm{La}$ in $\mathrm{Ba}_{2} \mathrm{SnO}_{4}$.

In present investigation the synthesis of $\mathrm{La}$ doped at the site of $\mathrm{Ba}_{2} \mathrm{SnO}_{4}$ have been carried out using traditional ceramic route by calcining at $1000^{\circ} \mathrm{C}$. The phase identification of the synthesized samples is confirmed using X-ray diffraction (XRD) followed by Rietveld refinement. The solubility limit of $\mathrm{La}$ in $\mathrm{Ba}_{2} \mathrm{SnO}_{4}$ lattice has been investigated by Raman spectroscopy and Fourier transform infrared spectroscopy (FTIR). The morphological and electrical properties of sintered ceramics were further studied using a field emission scanning electron microscope and a high precision LCR meter respectively.

\section{Experimental:}

The different compositions of $\mathrm{Ba}_{2-\mathrm{x}} \mathrm{La}_{x} \mathrm{SnO}_{4}$ (where $\mathrm{x}=0,0.01,0.02,0.04,0.06,0.10$ ) have been prepared via traditional ceramic route using $\mathrm{BaCO}_{3}, \mathrm{SnO}_{2}$, and $\mathrm{La}_{2} \mathrm{O}_{3}$ as raw materials. The composition with different doping concentration was referred by abbreviated names; B2LS0 for $\mathrm{x}=0$ and B2LS1 for $\mathrm{x}=0.01$ and so on. For example, B2LS2 refers to the composition $\mathrm{Ba}_{1.98} \mathrm{La}_{0.02} \mathrm{SnO}_{4}$. The raw materials have been weighed in stoichiometric ratio and mixed using acetone as mixing media with constant speed of rotation $200 \mathrm{rpm}$ for $8 \mathrm{~h}$ in a ball - miller (Retsch PM-200). These prepared mixtures have been dried into an oven for $4 \mathrm{~h}$, then transferred to an alumina crucible and heated at $1000^{\circ} \mathrm{C}$ for $6 \mathrm{~h}$ in ambient atmosphere. Temperature of the muffle furnace was increased at a constant heating rate of $5^{\circ} \mathrm{C} / \mathrm{min}$. The obtained mixture powder was evenly mixed with $2 \%$ polyvinyl alcohol (PVA) and pressed into cylindrical pellets with a diameter of $12 \mathrm{~mm}$ and a thickness of $\approx 2 \mathrm{~mm}$ using a uniaxial hydraulic press. The pellets have been air sintered into a muffle furnace; intially the temperature was increased to $200^{\circ} \mathrm{C}$ with constant heating rate of $2^{\circ} \mathrm{C} / \mathrm{min}$ and hold for $1 \mathrm{~h}$ to complete burn off the binder. Then, the temperature of furnace increased to $1250^{\circ} \mathrm{C}$ with constant heating rate of $5^{\circ} \mathrm{C} / \mathrm{min}$ and hold for $6 \mathrm{~h}$. Thereafter, the furnace was normally cooled to room temperature. The sintered pellets were coated by conducting silver paste and firing at $400^{\circ} \mathrm{C}$ for $35 \mathrm{~min}$ to make perfect connection. Further, the silver coated pellets were projected for electrical properties measurement.

\section{Characterization techniques:}

The thermal analysis of prepared mixture was performed using thermogravimetric and differential scanning calorimetr (TGA/DSC) (Mettler Toledo) system from temperature 25$1000{ }^{\circ} \mathrm{C}$ at a constant heating rate of $10^{\circ} \mathrm{C} / \mathrm{min}$ in the presence of nitrogen gas. Crystal structure 
and phase analysis of prepared samples were studied using Rigaku Miniflex II desktop, X-ray diffractometer (XRD) using $\mathrm{Cu}-\mathrm{K} \alpha$ radiation as source obtained at $30 \mathrm{kV}$ and $20 \mathrm{~mA}$ and $\mathrm{Ni}$ as filter. The XRD patterns were collected within angular range of $20 \leq 2 \theta \leq 80$ at a constant step size $\Delta 2 \theta=0.02^{\circ}$. The Fourier transform infrared (FTIR) spectra of obtained samples were recorded by Fourier Transform Infrared Spectrometer (Shimadzu, Model DF 803) using KBr pellet method within the wave number range of $400-4000 \mathrm{~cm}^{-1}$. Raman spectra of prepared samples were recorded at room temperature using Raman spectrometer (Renishaw) equipped with a laser diode having wavelength $532 \mathrm{~nm}$. To record the Raman spectra, the sample was focused by incident laser beam at a 50× long distance objective attached to the Leica DM 2500 M microscope. The optical properties of prepared samples were studied using UV-visible spectrophotometer (UV-2600, Shimandzu) from wavelength 200 to $800 \mathrm{~nm}$ in the absorption mode. The scanning electron micrograph (SEM) of the fractured surface of sintered pellets were recorded by a scanning electron microscope (JEOL JSM 840 A). Further, the electrical properties of sintered ceramics were measured using high precision LCR meter (Agilent E$4980 \mathrm{~A})$ in a wide range of temperature $\left(300-600^{\circ} \mathrm{C}\right)$ and frequency $(20 \mathrm{~Hz}-2 \mathrm{MHz})$.

\section{Results and Discussion}

\subsection{Determination of calcination temperature}

The thermogravimetric (TG) and differential scanning calorimetry (DSC) analyses of B2LS0 and B2LS2 were carried out to determine the calcination temperature for the prepared mixture of samples, as shown in Fig. 1 (a) and (b), respectively. The TG curve of B2LS0 shows weight loss of $9 \%$ in a single step $\left(700{ }^{\circ} \mathrm{C}-1000{ }^{\circ} \mathrm{C}\right)$ whereas, B2LS2 shows weight loss of $17.08 \%$ in two steps; first of $6.37 \% 25^{\circ} \mathrm{C}$ to $100{ }^{\circ} \mathrm{C}$ and the second of $10.71 \%$ from $100{ }^{\circ} \mathrm{C}$ to 1000 ${ }^{\circ} \mathrm{C}$. Due to the limitations of the instruments used in the investigation, the TG/DSC data could not be recorded above $1000^{\circ} \mathrm{C}$. The reaction mechanism of $\mathrm{Ba}_{2} \mathrm{SnO}_{4}$ was well described in literature and suggests that the reaction between the raw materials takes place by equation given below [15];

$2 \mathrm{BaCO}_{3}+\mathrm{SnO}_{2} \rightarrow \mathrm{Ba}_{2} \mathrm{SnO}_{4}+2 \mathrm{CO}_{2}$

The primary difference between the TGA curve of B2LS0 and B2LS2 is additional weight loss of $6.37 \%$ in B2LS2, which may be attributed to the hygroscopic aspect of $\mathrm{La}_{2} \mathrm{O}_{3}$ [31]. The DSC curve of each sample shows an endothermic peak present at $828^{\circ} \mathrm{C}$ and $830^{\circ} \mathrm{C}$ in sample B2LS0 and B2LS2 respectively. The appearance of an endothermic peak in each sample 
indicates the initiation of a reaction between the raw materials. The reaction between the reactants is expected to proceed in the same manner as B2LS0;

$$
1.98 \mathrm{BaCO}_{3}+0.01 \mathrm{La}_{2} \mathrm{O}_{3}+\mathrm{SnO}_{2} \rightarrow \mathrm{Ba}_{1.98} \mathrm{La}_{0.02} \mathrm{SnO}_{4}+1.98 \mathrm{CO}_{2}+0.005 \mathrm{O}_{2} \uparrow
$$

The weight loss has been calculated from equation (1) and (2) are almost $16.03 \%$. The weight loss occurred up to $100{ }^{\circ} \mathrm{C}$ in B2LS2 is assigned to the evaporation of water molecules adsorbed at the surface of particles that adsorbed during the mixing of solids [32]. Since CO2 loss is the primary cause of weight loss in both samples, and the total weight loss obtained experimentally is nearly half of what is predicted. The huge difference between experimental and theoretical weight loss suggesting that the decomposition of $\mathrm{BaCO}_{3}$ does not takes place completely because the reaction not only depends on temperature but also on time. Furthermore, the presence of a weak endothermic reaction peak about $830{ }^{\circ} \mathrm{C}$ in the DSC curves of both samples suggests that the reaction begins before complete decomposition of $\mathrm{BaCO}_{3}$. The presence of reaction peak was in well agreement with the earlier study [33]. Since the amount of doping was quite low, so no significant changes have been observed in present investigation except the hygroscopic properties of La. Therefore, to obtain the phase pure powder the mixture of raw materials should be calcined at $1000^{\circ} \mathrm{C}$ for $6 \mathrm{~h}$.
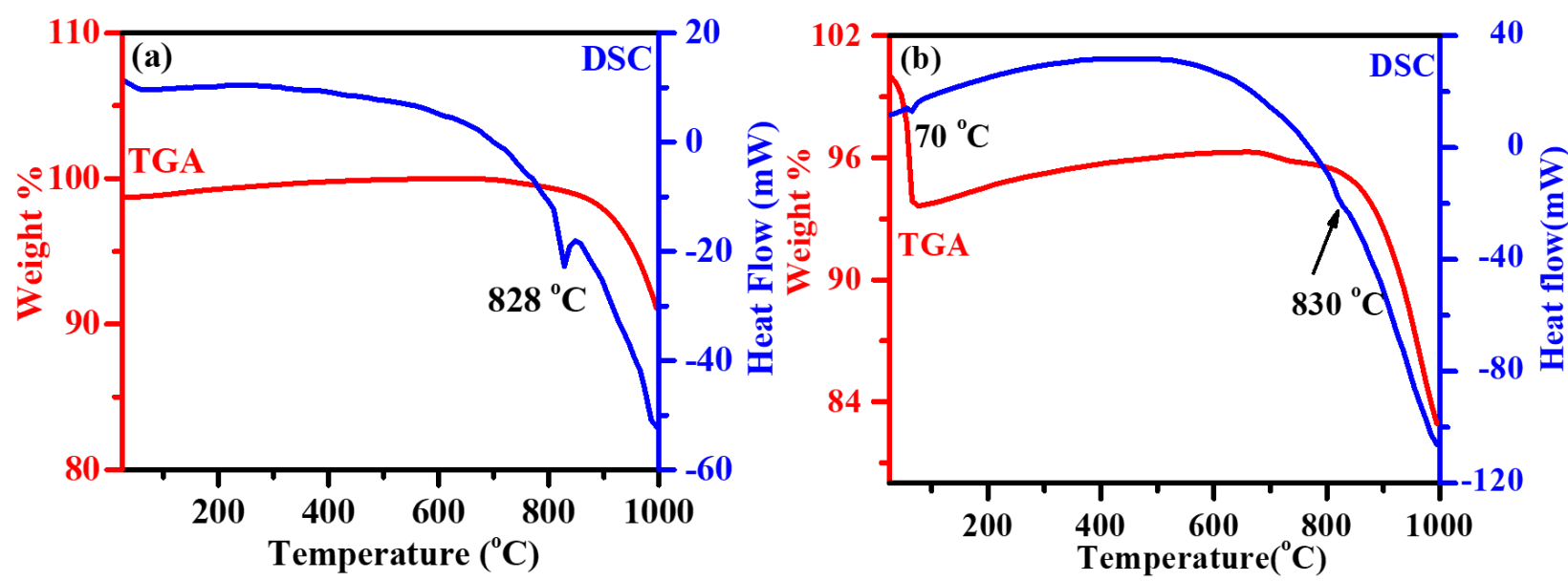

Fig. 1: Thermogravimetric and differential scanning calorimetry curve of prepared mixture of sample (a) B2LS0, (b) B2LS2.

\subsection{Determination of phase and crystal structure:}

The phase and crystal structure of the prepared samples were determined using powder X-ray diffraction (XRD) analysis. The XRD pattern of calcined powders has been recorded within the angular range $25-80^{\circ}$ and shown in Fig. 2. All the diffraction peaks observed in XRD pattern are fine and sharp, suggesting that the sample is crystalline. In order to determine the phase of the sample, the experimental XRD pattern is compared to the JCPDS database and found to be 
very similar to the JCPDS file (no- 43-0071) of parent phase $\mathrm{Ba}_{2} \mathrm{SnO}_{4}$ [34]. It has also been observed that as La is incorporated into the lattice, the size and width of the peaks gradually alter. A new XRD peak (denoted by *) at $27.72^{\circ}$, which belongs to the pyrochlore phase $\mathrm{La}_{2} \mathrm{Sn}_{2} \mathrm{O}_{7}$ (JCPDS card \#73-1686), is observed as the concentration of La increased above $\mathrm{X}=0.04$. The other pyrochlore phase XRD peaks are located at $35.12^{\circ}, 51.88^{\circ}$, and $62.46^{\circ}$, which are almost identical to the XRD peak of $\mathrm{Ba}_{2} \mathrm{SnO}_{4}$. The intensity of this peak steadily increases, with increasing La. The amount of pyrochlore phase has been estimated using the reference intensity ratio method and found to be approximately $2 \%$ for sample B2LS6 and $6 \%$ for sample B2LS10. This means that the solubility of $\mathrm{La}$ at the $\mathrm{Ba}$ site of $\mathrm{Ba}_{2} \mathrm{SnO}_{4}$ is between 0.04-0.06 weight $\%$. $\mathrm{Ba}_{2} \mathrm{SnO}_{4}$ is made up of a perovskite $\mathrm{BaSnO}_{3}$ and a rock salt layer of $\mathrm{BaO}$, meaning that the number of $\mathrm{Ba}$ sites is greater than that of $\mathrm{BaSnO}_{3}$. The solubility limit of $\mathrm{La}$ is found to be close to the La-doping in alkaline perovskite oxides [30,31,35]. This indicates that while there are more sites in $\mathrm{Ba}_{2} \mathrm{SnO}_{4}$ than in $\mathrm{BaSnO}_{3}$, the $\mathrm{La}$ is only occupying the sites that are available in $\mathrm{BaSnO}_{3}$. In order to understand such behaviour theoretical study must be needed. 


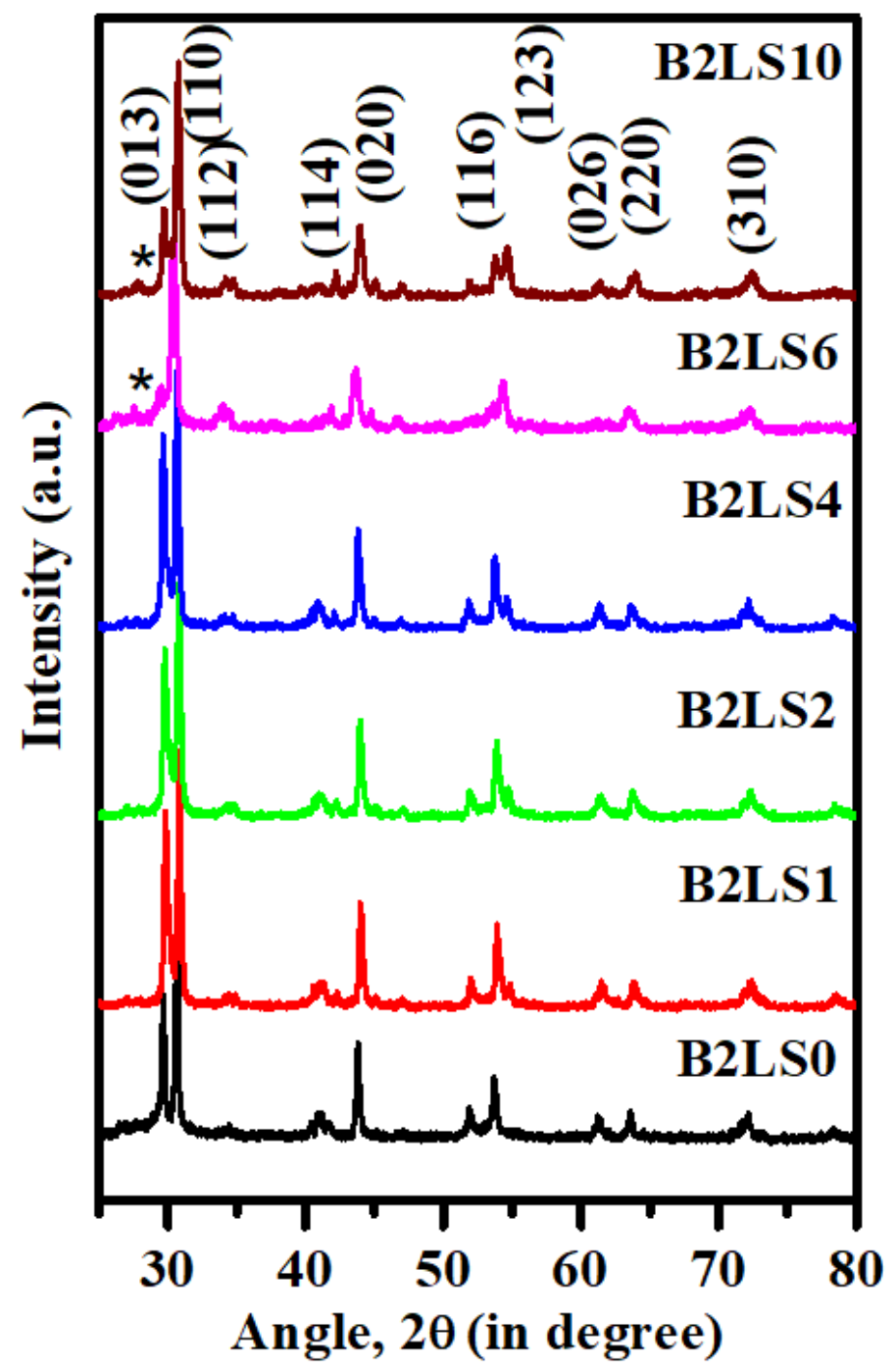

Fig. 2: X-ray diffraction (XRD) pattern of all samples obtained at room temperature.

\subsection{Fourier transform infrared (FTIR) Spectroscopy analysis:}

Fourier transform infrared (FTIR) spectrum analysis is used to identify the presence of various functional groups in samples, as shown in Fig. 3 (a). The FTIR spctra of all samples are similar to the FTIR spectra of pure $\mathrm{Ba}_{2} \mathrm{SnO}_{4}$ reported in literature [22]. The FTIR spectra shows bands at $518 \mathrm{~cm}^{-1}, 623 \mathrm{~cm}^{-1}, 850 \mathrm{~cm}^{-1}, 1020 \mathrm{~cm}^{-1}, 1122 \mathrm{~cm}^{-1}, 1419 \mathrm{~cm}^{-1}, 1952 \mathrm{~cm}^{-1}$ and a wide band 2424-3645 $\mathrm{cm}^{-1}$ in B2LS0. However, the addition of La to the sample suppressed a few of the bands $\left(1020,1122,1952 \mathrm{~cm}^{-1}\right)$ and moved the other bands to higher wavenumbers. The vibrations of $\mathrm{BaO}$ and $\mathrm{SnO}_{6}$ octahedra are assigned to the two bands present at $515 \mathrm{~cm}^{-1}$ and $616 \mathrm{~cm}^{-1}$, respectively $[32,36]$. The expand view of the characteristic band has been shown in Fig. 3 (b) in order to describe the incorporation of La into the lattice. The bands present at 515 $\mathrm{cm}^{-1}$ shifts towards higher wavenumber while the position of $623 \mathrm{~cm}^{-1}$ unchanged with incorporation of La. In terms of effective reduced mass and bond length, this difference can be 
explained. The following equation demonstrates a straightforward understanding of this variance;

$\vartheta=\frac{1}{2 \pi} \sqrt{\frac{K}{\mu}}$

Where $K$ is a constant considered to be as effective bond length and $\mu$ is the reduced mass of band arrangement. Since La has a higher mass than $\mathrm{Ba}$, the reduced mass decreases as La is incorporated, resulting in a higher wavenumber needed to vibrate the specific band. Besides these characteristics bands, few more bands are observed at 856,1024, 1120, 1450, $1936 \mathrm{~cm}^{-1}$ assigned to the physically adsorbed $-\mathrm{CO}_{2}$ and the bands at 1936 and $3294 \mathrm{~cm}^{-1}$ is assigned to the adsorbed $-\mathrm{OH}$ at the surface of the sample [36]. It is noticed that with increasing the concentration of $\mathrm{La}$ at the $\mathrm{Ba}$ site above $\mathrm{x}=0.04$, a weak XRD peak belongs to phase $\mathrm{La}_{2} \mathrm{Sn}_{2} \mathrm{O}_{7}$ is appeared in XRD pattern. However, the vibrational band associated with $\mathrm{La}_{2} \mathrm{Sn}_{2} \mathrm{O}_{7}$ is observed within the wavenumber range 550 to $680 \mathrm{~cm}^{-1}$ [37]. So, the identification of the pyrochlore phase present in the sample can't be done using this analysis. Therefore, the identification of pyrochlore phase is further analyzed using Raman spectroscopy and discussed in susequent section.

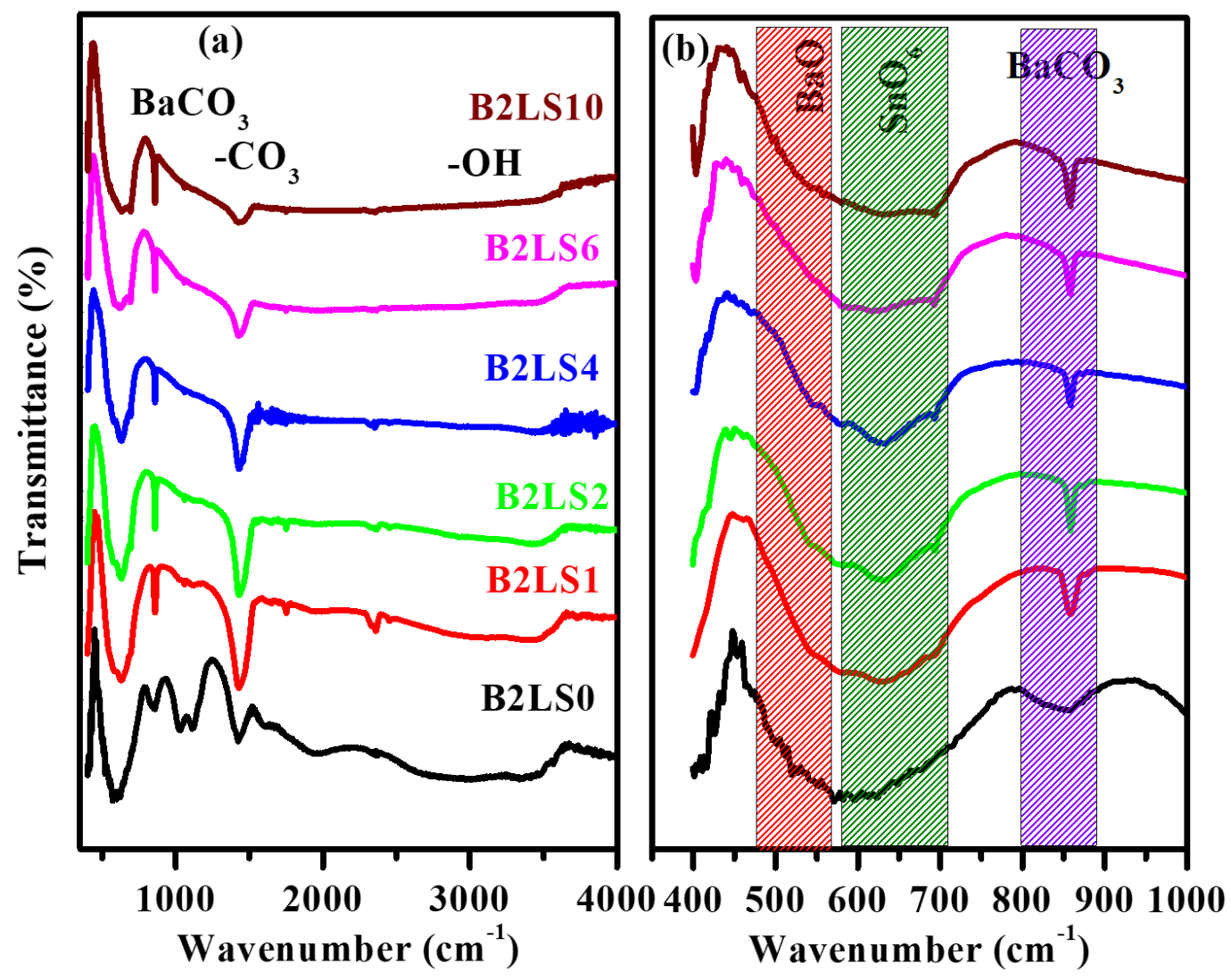

Fig. 3 (a) Fourier transform infrared (FTIR) spectra of prepared samples (b) Enlarge view of FTIR spectra obtained within $400-1000 \mathrm{~cm}^{-1}$. 


\subsection{Raman Spectroscopy analysis:}

Raman spectroscopy is a novel non-destructive and more sensitive technique in comparison with XRD, that usually revealed the local structural information of material [36]. It has piqued interest because of its ability to detect structural changes that alter crystal symmetry at the submicron scale, as well as provide an estimation of the factors that cause these structural changes. Fig. 4 illustrates the Raman spectrum of all samples recorded at room temperature within the wavenumber range $100-1000 \mathrm{~cm}^{-1}$. Raman spectrum of the synthesized samples is compared with the Raman spectrum of $\mathrm{K}_{2} \mathrm{NiF}_{4}$ type layered perovskite materials [15]. Theoretical calculation revealed that the $\mathrm{A}_{2} \mathrm{BO}_{4}$ type structures have total 14 optical modes, and among them only 4 modes were found to be Raman active. In $\mathrm{A}_{2} \mathrm{BO}_{4}$ type structure, $\mathrm{B}$ atom was shared by 6 oxygen (4 equatorial oxygen $\mathrm{O} 1$ under $\mathrm{D}_{2 \mathrm{~h}}$ symmetry and two apical oxygen $\mathrm{O} 2$ under $\mathrm{C}_{4 v}$ symmetry) while 9 oxygens were shared by atom $\mathrm{A}$. The rock salt A-O is connected with the perovskite through apical oxygen along c-direction. The $\mathrm{AO}_{9}$ dodecahedral involves four equatorial oxygen $\mathrm{O} 2$ at same position $\mathrm{Z}$ and one apical oxygen along c direction and also four $\mathrm{O} 1$ at $\mathrm{z}=0$ as shown in Fig. 4. The 14 optical modes have been obtained by the contribution of phonon associated with various atoms are given below;

$A \rightarrow C_{4 v} \rightarrow A_{1 g}+E_{g}+A_{2 u}+E_{u}$

$B \rightarrow D_{4 h} \rightarrow A_{2 u}+E_{u}$

$O(1) \rightarrow D_{2 h} \rightarrow A_{2 u}+B_{2 u}+2 E_{u}$

$O(2) \rightarrow C_{4 v} \rightarrow A_{1 g}+E_{g}+A_{2 u}+E_{u}$

$\Gamma=2 A_{1 g}+2 E_{g}+4 A_{2 u}+5 E_{u}+B_{2 u}$

Among 14 optical modes only four modes are Raman active (two $\mathrm{A}_{1 \mathrm{~g}}$ and two $\mathrm{E}_{\mathrm{g}}$ ), while experimentally more than four modes are found to be active in present samples $[15,38]$. The various Raman bands observed in the synthesized sample are assigned to different modes and listed in Table 1. The band appeared at $562 \mathrm{~cm}^{-1}$ is characteristic band, that present due to the vibration of oxygen atom within the different plane such as $\mathrm{Ox}, \mathrm{Oy}$, and Oz. The nature of this band showing slight asymmetry due to crystal structure of $\mathrm{Ba}_{2} \mathrm{SnO}_{4}(a=b \neq c)$. This characteristic band shows systematic shift towards higher wavenumber due to variation in structural parameters and effective mass. The structural characterization section shows that the substitution of La compressed the all three directions and decreases the net reduced mass. So, this may be a possible reason to observed the shift in position of Raman band towards higher wavenumber (See equation (3)). It is further noticed that a new Raman band is appeared at 597 $\mathrm{cm}^{-1}$ for samples B2LS6 and B2LS10 because of the presence of pyrochlore phase $\left(\mathrm{La}_{2} \mathrm{Sn}_{2} \mathrm{O}_{7}\right)$ 
present in sample. The Raman study is completely agreed with XRD analysis, that further reconfirm that the solubility $\mathrm{La}$ is limited into the lattice between $0.04<\mathrm{x} \leq 0.06$ weight $\%$.

Table 1: The assignment of different Raman Bands observed in Fig. 5.

\begin{tabular}{|c|c|c|c|c|c|c|c|c|}
\hline \multirow{2}{*}{$\begin{array}{l}\text { Peak } \\
\text { notation }\end{array}$} & \multicolumn{6}{|c|}{ Peak position $\left(\mathrm{cm}^{-1}\right)$} & \multirow[t]{2}{*}{ Assignment } & \multirow{2}{*}{$\begin{array}{l}\text { In } \\
\text { Fig. } \\
5 a\end{array}$} \\
\hline & B2LS0 & B2LS1 & B2LS2 & B2LS4 & B2LS6 & B2LS10 & & \\
\hline \multirow[t]{2}{*}{$E_{g}$} & 133.20 & 136.20 & 134.73 & 136.59 & 147.81 & 147.81 & $\begin{array}{l}\text { Vibration of Ba- } \\
\mathrm{O} 2 \text { in ab plane }\end{array}$ & $v_{1}$ \\
\hline & 150.62 & 155.87 & 164.80 & 155.87 & 177.22 & 179.89 & $\begin{array}{l}\text { Vibration of Ba- } \\
\text { O1 in ab plane }\end{array}$ & $v_{1}^{\prime}$ \\
\hline$A_{1 g}$ & 194.65 & 202.15 & 227.22 & 188.65 & 209.18 & 254.22 & $\begin{array}{l}\text { Vibration of Ba- } \\
\mathrm{O} 2 \text { along c-axis }\end{array}$ & $v_{2}$ \\
\hline \multirow[t]{3}{*}{$E_{g}$} & 299.18 & 304.36 & 293.06 & 296.65 & 252.89 & 338.27 & $\begin{array}{l}\text { Vibration of } \mathrm{O} 2 \text { of } \\
\mathrm{SnO}_{6} \text { octahedra in } \\
\text { ab plane }\end{array}$ & $v_{3}$ \\
\hline & 331.18 & 333.29 & 327.07 & 333.29 & & 401.99 & $\begin{array}{l}\text { Vibration of } \\
\text { bridging oxygen in } \\
\text { Ba-O2-Ba in ab- } \\
\text { plane }\end{array}$ & $v_{3}^{\prime}$ \\
\hline & 440.71 & 443.21 & 456.45 & 449.00 & 472.87 & 472.87 & Second order band & $*$ \\
\hline \multirow[t]{3}{*}{$A_{1 g}$} & 559.46 & 564.71 & 565.92 & 562.78 & 572.55 & 578.55 & $\begin{array}{l}\text { Vibration of } \\
\text { oxygen O2 of } \\
\text { SnO6 along c-axis }\end{array}$ & $v_{4}$ \\
\hline & & & & & 597.49 & 597.49 & $\begin{array}{l}\text { Vibration of } \\
\mathrm{La} 2 \mathrm{Sn} 2 \mathrm{O} 7\end{array}$ & $@$ \\
\hline & 687.46 & 691.89 & 683.72 & 691.98 & 700.14 & 698.90 & $\begin{array}{l}\text { Presence of } \mathrm{BaCO}_{3} \\
\text { at the surface }\end{array}$ & * \\
\hline
\end{tabular}




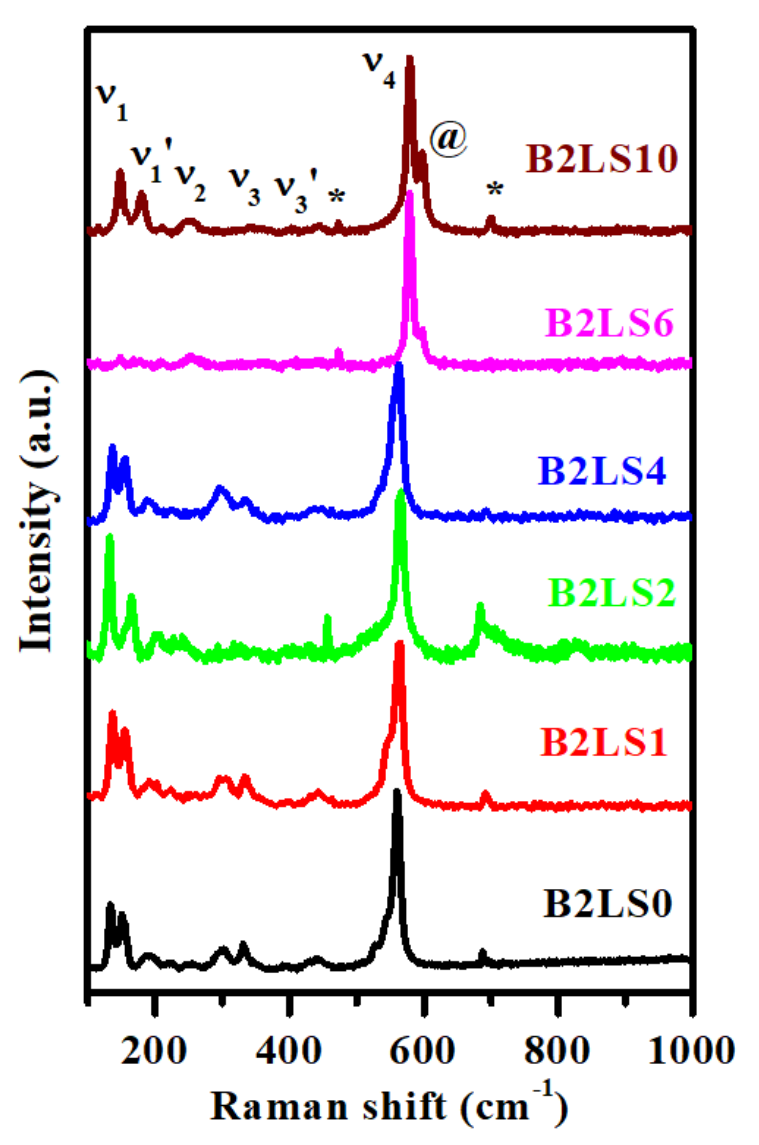

Fig. 4: Raman spectra of prepared samples obtained at room temperature.

\subsection{UV-absorption spectroscopy:}

The UV absorption spectrum of all the compositions have been recorded within the wavelength range 200-800 $\mathrm{nm}$ and shown in Fig. 5. The spectrum shows an intense absorption in UVregion (370-440 nm). The value of absorption wavelength was determined by extrapolating their absorption to the wavelength axis and found to be $375 \mathrm{~nm}, 392 \mathrm{~nm}, 394 \mathrm{~nm}, 430 \mathrm{~nm}, 399$ $\mathrm{nm}$ and $395 \mathrm{~nm}$ respectively for samples B2LS0, B2LS1, B2LS2, B2LS4, B2LS6 and B2LS10. With the addition of La, the absorption peak shifts to a higher wavelength, which may be due to an inter-shell transition from the energy level of La [36]. The magnitude of absorption varies with La within the UV-region, which could be utilized as metastable state for UV-detector application. 


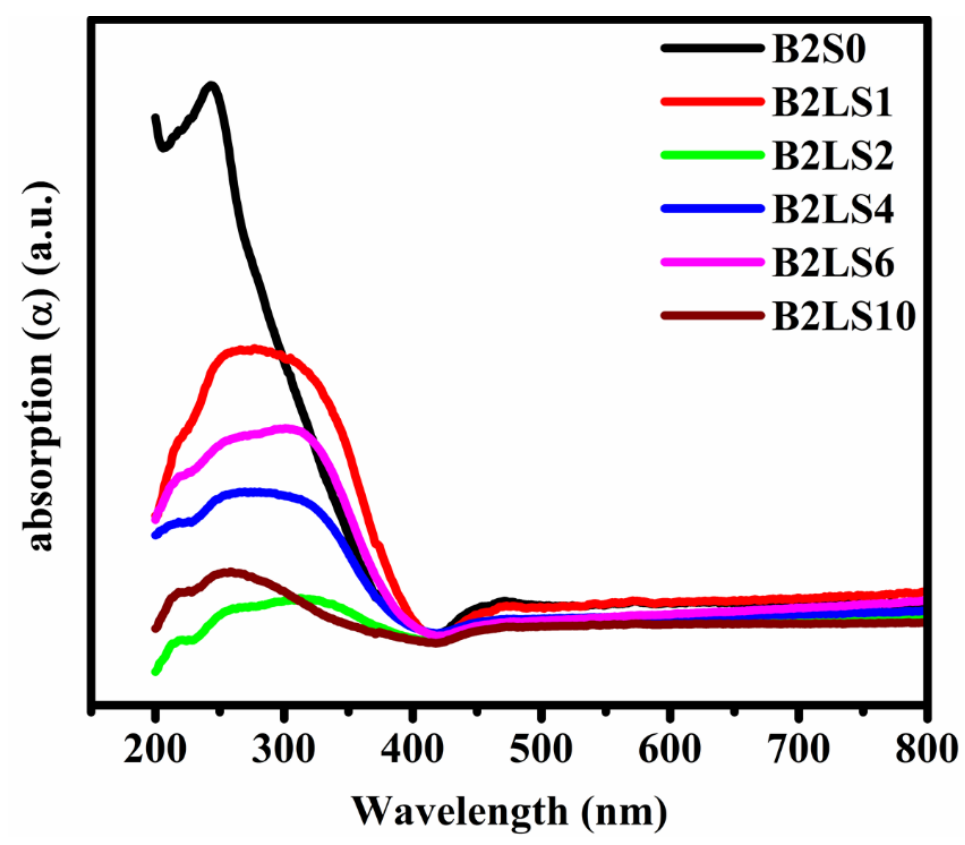

Fig. 5: Ultra-violet (UV) vis spectra of all samples obtained at room temperature.

The optical band gap of the samples is determined from UV absorption data using Tauc relation given by [39]:

$$
(\alpha h v)=B\left(h v-E_{g}\right)^{m}
$$

Where $\alpha$ is the absorption coefficient, $h v$ is the energy of photon, B is an energy independent constant, $E_{g}$ is the energy band gap of the material and $m$ is known as index parameter, which decides the nature of transition. Based on available literature, four values of $m$ is possible such as $2,3 / 2,1$, and $1 / 2$ for indirect allowed (indirect band gap) and indirect forbidden, direct forbidden and direct allowed (direct band gap) electronic transition respectively [40]. The direct band gap results due to transition of photon from the bottom of conduction band to top of valence band whereas indirect band gap results due to transition of photon from the intermediate energy states such as defects [41]. The Tauc plot is generated using equation (4) for both direct and indirect band gap of the samples by plotting $(\alpha h v)^{2}$ and $(\alpha h v)^{1 / 2}$ at y-axis and energy of photon (hv) at x-axis and shown in Fig. 6. Extrapolating the linear portion of the curve to the $\mathrm{x}$-axis provides the direct and indirect band gap values. The values of direct band gap for undoped sample obtained in this work is in agreement with the values reported in the literature [42,43]. The value of direct bandgap was found to be $3.75 \mathrm{eV}, 3.23 \mathrm{eV}, 3.28 \mathrm{eV}$ and $3.30 \mathrm{eV}$ and indirect bandgap to be $3.26 \mathrm{eV}, 2.92 \mathrm{eV}, 3.04 \mathrm{eV}$ and $3.05 \mathrm{eV}$ for samples B2LS0, B2LS1, B2LS2 and B2LS4 respectively. However, as XRD and Raman analysis indicate that the presence of pyrochlore phase in sample B2LS6 and B2LS10, the UV-Vis spectra also 
reflect two bandgap one is may be of B2LS composition and other for pyrochlore phase. In present case, the discussion for sample B2LS6 and B2LS10 excluded due to presence of pyrochlore phase and solubility limit of La into the lattice. One major change has been observed that the direct bandgap and indirect bandgap of the La-doped samples are found to be lower than undoped. In order to understand this variation, the charge compensation mechanism has been taken into account. Since La acts as donor for Ba-site and the charge compensation mechanism takes place by following equation;

$\mathrm{La}_{2} \mathrm{O}_{3} \stackrel{\mathrm{Ba}_{2} \mathrm{SnO}_{4}}{\longrightarrow} 2 \mathrm{La}_{\mathrm{Ba}}+\mathrm{Sn}_{\mathrm{Sn}}^{x}+6 \mathrm{O}_{O}^{x}+V_{\mathrm{Ba}}^{\prime \prime} / 2 e^{\prime}$

Since the samples are synthesized at high temperature, so the loss of oxygen can't be ruled out and given by;

$O_{o} \rightarrow \frac{1}{2} O_{2}(g)+\ddot{V}_{O}+2 e^{\prime}$

The position of defect state $2 L \dot{a}_{B \boldsymbol{a}}$ formed below the conduction band that might be one of the possible reasons for decreasing the bandgap. However, as La is increased, the concentration of defect state $2 L \dot{a}_{\boldsymbol{B a}}$ increases, since this defect has a positive charge and as its concentration increases, a small amount of columbic repulsive force between defect states acts, which may be an explanation for the bandgap rising. Moreover, the presence of indirect bandgap in samples suggests the presence of defect such as oxygen vacancy (see equation $(6)$ ) $[33,44]$. Since the difference between the direct and indirect bandgap has been found to be $0.49 \mathrm{eV}$, $0.31 \mathrm{eV}, 0.24 \mathrm{eV}$ and $0.25 \mathrm{eV}$ for samples B2LS0, B2LS1, B2LS2 and B2LS4 respectively. Since the value of difference has been decreased with La, that might be due to increase of oxygen vacancy. The value of direct bandgap reflects inorganic semiconducting nature of samples, and utilized it for semiconductor device application. 

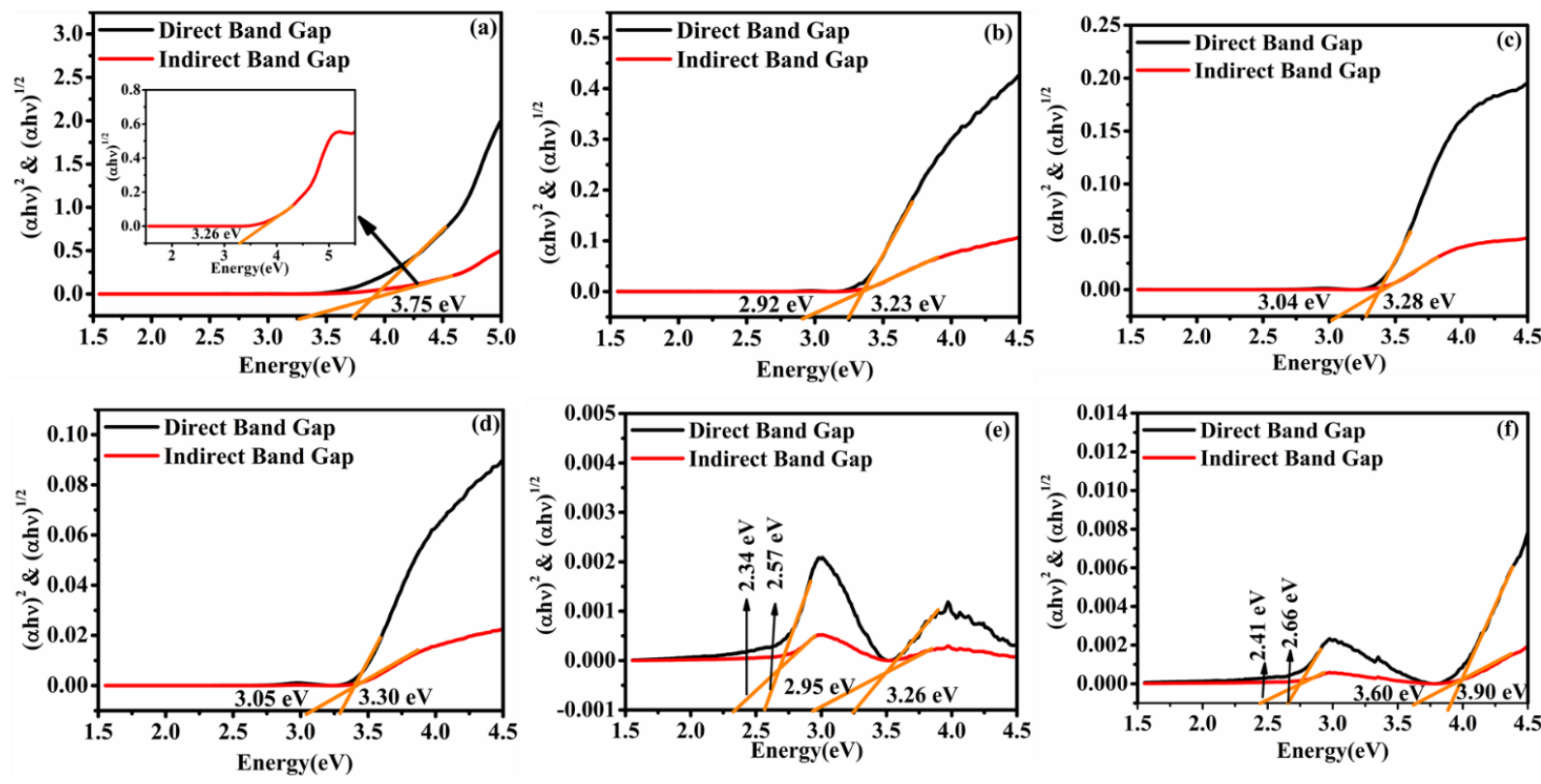

Fig. 6: Tauc's plot generated for direct and indirect band gap of all samples.

\subsection{Morphological studies of sintered pellets of system (Ba1-xLax)2 $)_{2} \mathrm{SnO}_{4}$}

To study electrical and morphological properties of the synthesized samples, calcined powders of all the compositions have been pelletized and sintered at $1250{ }^{\circ} \mathrm{C}$ for $6 \mathrm{~h}$. For microstructural studies freshly fractured surfaces of the sintered pellets were coated with gold using sputtering unit. The Field emission scanning electron micrograph (FESEM) of the fractured surface of the samples have been recorded and shown in Fig. 7. From Fig. 7, it has been noticed that the grains are agglomerated and spherical in shape. However, the degree of agglomeration among the grains reduces with La. Further, the value of average grain size for all samples have been calculated using 'Image J' software. Initially, the histogram between the number of grain and their size has been generated for all samples and shown in the inset of each figure of Fig. 7. In order to determine the average grain size, the Gaussian distribution function has been fitted to the histogram and the value of grain size is given in the Fig 7. It has been further noticed that the value of average grain size is decreased with increasing concentration of dopant. Since $\mathrm{La}^{3+}$ at $\mathrm{Ba}^{2+}$ site of $\mathrm{Ba}_{2} \mathrm{SnO}_{4}$ act as a donor and charge neutrality may be maintained by equation (5). As the concentration of $\mathrm{La}$ in the $\mathrm{Ba}_{2} \mathrm{SnO}_{4}$ increases the concentration of either electron or $V_{B a}^{\prime \prime}$ increases. It is well reported in literature that the donors act as grain growth inhibitor whereas oxygen vacancies promotes the grain growth process [45]. This might be possible reason for the variation of grain size observed in present samples. 


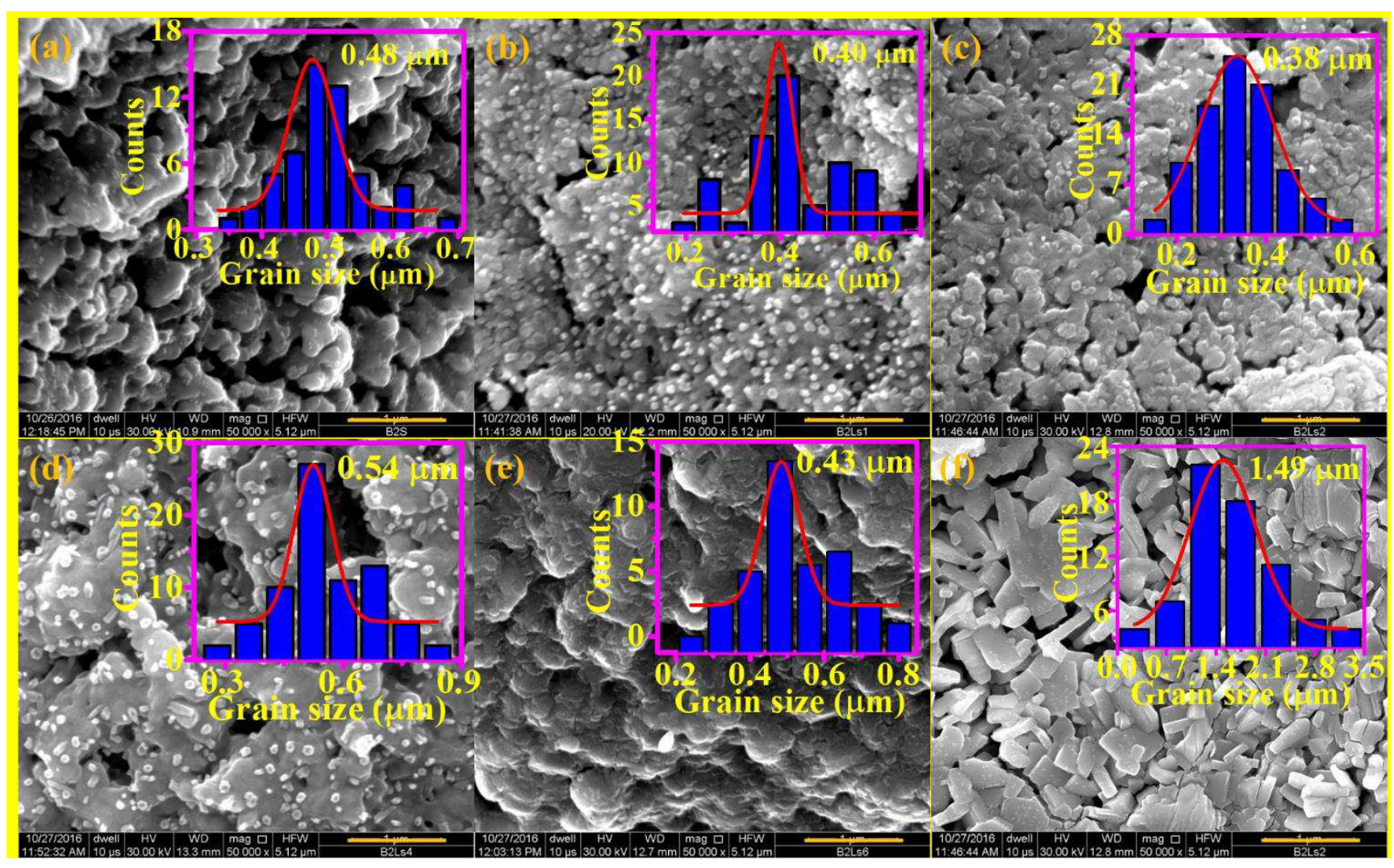

Fig. 7: Field Emission Scanning Electron Micrograph (FESEM) of the fractured surface of sintered samples (a) B2LS0 (b) B2LS1 (c) B2LS2 (d) B2LS4 (e) B2LS6 (f) B2LS10.

\subsection{Characterization of Single-phase powders of $\left(\mathrm{Ba}_{1-\mathrm{x}} \mathrm{La}_{\mathrm{x}}\right)_{2} \mathrm{SnO}_{4}$ system}

\subsubsection{Reitveld profile analysis:}

Structural parameters of the single-phase samples have been determined using Reitveld refinement analysis performed on powder X-ray diffraction data. The refinement has been performed using FullProf software suite by adopting crystal structure as tetragonal with space group (\#139, I4/mmm). The peak function has been modelled as Pseudo-Voight (A combination of Lorentzian and Gaussian function) given by;

$P . V .=\eta * L+(1-\eta) * G$

Where, $\eta, L, G$ are the positive constant, contribution of Lorentzian and Gaussian function present in XRD peak respectively. The background for the XRD diffraction pattern has been described in terms of $12^{\text {th }}$ order polynomial function [46]. The refinement has been processed until the achievement of best fit. The Reitveld refined pattern of single-phase solid solutions are shown in Fig. 8(a). The quality of fitting has been judged by calculating a parameter $\mathrm{S}$ using weighted profile pattern $\left(\mathrm{R}_{\mathrm{wp}}\right)$ and pattern profile $\left(\mathrm{R}_{\mathrm{p}}\right)$ through formula, $S=R_{w p} / R_{p}$. The value of $S$ for all samples found to be unity suggests that the parameters derived from 
refinements are relevant. The refined parameters along with other relevant structural parameters such as most affected bond length and bond angles are given in Table 2.

Table 2: Lattice Parameters, Position parameters, Refinement parameters, Bond length, Bond angle, Crystallite size and micro-strain of all samples.

\begin{tabular}{|c|c|c|c|c|}
\hline Parameters & B2LS0 & B2LS1 & B2LS2 & B2LS4 \\
\hline \multicolumn{5}{|c|}{ Lattice Parameters } \\
\hline$a=b$ & 4.1458 & 4.1419 & 4.1418 & 4.1413 \\
\hline $\mathrm{c}$ & 13.2899 & 13.2950 & 13.2859 & 13.2915 \\
\hline Volume & 228.42 & 228.08 & 227.91 & 227.95 \\
\hline $\mathrm{X}$-ray density $\left(\mathrm{g} / \mathrm{cm}^{3}\right)$ & 7.53 & 7.036 & 6.68 & 6.59 \\
\hline \multicolumn{5}{|c|}{ Position Coordinate } \\
\hline $\mathrm{Ba}$ & $(0,0,0.35235)$ & $(0,0,0.35036)$ & $(0,0,0.35268)$ & $(0,0,0.34830)$ \\
\hline $\mathrm{La}$ & -- & $(0,0,0.35036)$ & $(0,0,0.35268)$ & $(0,0,0.34830)$ \\
\hline $\mathrm{Sn}$ & $(0,0,0)$ & $(0,0,0)$ & $(0,0,0)$ & $(0,0,0)$ \\
\hline $\mathrm{O} 1$ & $(0.5,0,0)$ & $(0.5,0,0)$ & $(0.5,0,0)$ & $(0.5,0,0)$ \\
\hline $\mathrm{O} 2$ & $(0,0,0.13636)$ & $(0,0,14111)$ & $(0,0,0.15390)$ & $(0,0,0.16091)$ \\
\hline \multicolumn{5}{|c|}{ Refinement Parameters } \\
\hline $\mathrm{Rp}$ & 10.5 & 10.6 & 12.5 & 8.3 \\
\hline Rwp & 11.8 & 11.4 & 13.1 & 9.6 \\
\hline $\operatorname{Re}$ & 13.03 & 8.4 & 9.01 & 9 \\
\hline$\chi^{2}$ & 3.72 & 5.5 & 6.1 & 5.6 \\
\hline $\mathrm{S}=\mathrm{R}_{\mathrm{wp}} / \mathrm{R}_{\mathrm{p}}$ & 1.12 & 1.07 & 1.05 & 1.15 \\
\hline \multicolumn{5}{|c|}{ Bond Length } \\
\hline $\mathrm{Ba}-\mathrm{Sn}$ & 3.5577 & 3.5275 & 3.5125 & 3.5053 \\
\hline $\mathrm{Ba}-\mathrm{O} 1$ & 2.8544 & 2.8495 & 2.8368 & 2.8302 \\
\hline $\mathrm{Ba}-\mathrm{O} 2$ & 2.9354 & 2.9341 & 2.9321 & 2.9303 \\
\hline Sn-O1 & 2.0729 & 2.0728 & 2.0709 & 2.0706 \\
\hline $\mathrm{Sn}-\mathrm{O} 2$ & 1.8122 & 2.1305 & 2.1447 & 2.1600 \\
\hline \multicolumn{5}{|c|}{ Bond Angle } \\
\hline O2-Ba-O2 (layer) & 176.14 & 176.74 & 176.88 & 177.00 \\
\hline $\begin{array}{l}\text { Ba-O1-Ba } \\
\text { (Perovskite) }\end{array}$ & 86.859 & 86.799 & 86.669 & 86.477 \\
\hline
\end{tabular}




\begin{tabular}{|l|l|l|l|l|}
\hline Crystallite size $(\mathrm{nm})$ & 24.47 & 18.84 & 17.52 & 16.48 \\
\hline Micro-Strain $\left(\times 10^{-3}\right)$ & 2.91 & 3.89 & 4.91 & 6.06 \\
\hline
\end{tabular}

From Table 2, it has been noticed that the value of lattice parameters $(a, b)$ found to be decrease while parameter c shows random variation. However, the volume of unit cell and x-ray density found to be decrease with incorporation of La into the lattice. Further, the different bond lengths $\mathrm{Ba}-\mathrm{Sn}, \mathrm{Ba}-\mathrm{O} 1, \mathrm{Ba}-\mathrm{O} 2$ and $\mathrm{Sn}-\mathrm{O} 1$ were also found to be decrease with La that supports the experimental results. The variation in these structural parameters were found due to difference in ionic radii of dopant $\mathrm{La}^{3+}(1.21 \AA)$ to host $\mathrm{Ba}^{2+}(1.44 \AA)$ [47]. The crystal structure of a reference sample has been generated using crystallographic information file (CIF) through the Vesta Software and shown in Fig. 8(b). In the crystal structure, the perovskite $\left(\mathrm{BaSnO}_{3}\right)$ is sandwiched between two adjacent rock salt layers $(\mathrm{BaO})$. The position coordinate of the atoms was already given in Table 2, that indicates that with incorporation of La, the z-coordinate of $\mathrm{Ba}$ and $\mathrm{O} 2$ shifted from their mean position. In this figure the number of unit cell along a,baxis is higher compared to c-axis, that might be a possible reason that the solubility of La at Ba-site takes place similar to the perovskite $\mathrm{BaSnO}_{3}$. As La occupied the sites available in lattice, the charge compensation mechanism takes place by equation (5) so the resultant charge may create lattice strain due to which small shift is observed in the position of $\mathrm{O} 2$ along $\mathrm{x}$-axis. This shift may apply a small amount of force on Ba present in layer and perovskite that could be seen from angle $(\mathrm{O} 2-\mathrm{Ba}-\mathrm{O} 2)$ and $(\mathrm{Ba}-\mathrm{O} 1-\mathrm{Ba})$. Further the effect of doping also studied in terms of micro-strain in subsequent section.
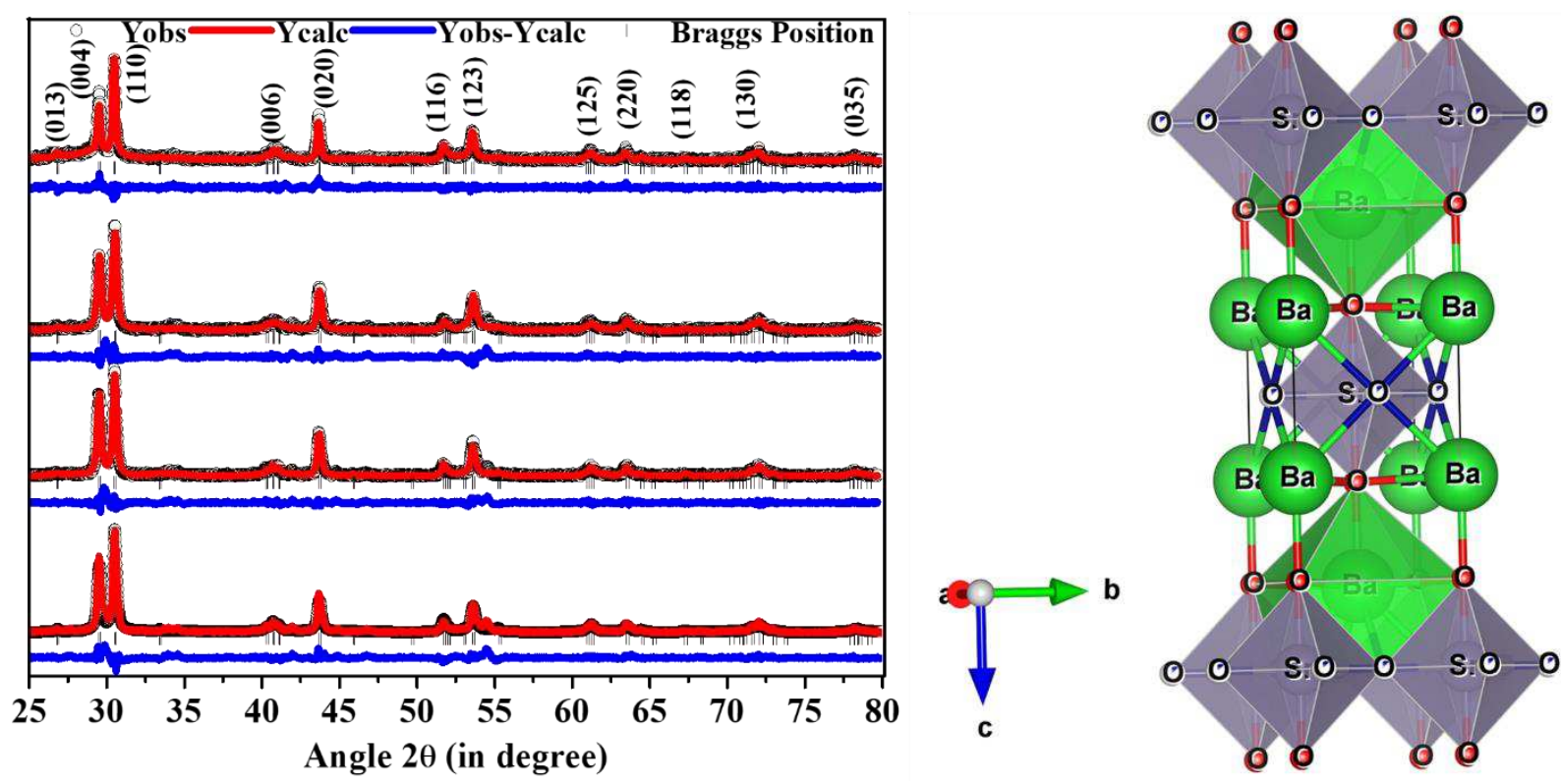
Fig. 8 (a) Rietveld refinement pattern of all samples, (Yobs, Ycalc, Yobs-Ycalc, and Bragg's position represent experimental pattern, calculated pattern, difference between experimental and calculated pattern, and Bragg's diffraction peaks respectively). (b) Crystal structure of a reference sample generated after refinement.

\section{Electrical Properties Measurement}

The electrical properties of silver coated pellets have been measured as a function of frequency $(20 \mathrm{~Hz}-2 \mathrm{MHz})$ and temperature range $300-600^{\circ} \mathrm{C}$. The frequency dependence conductivity for many polycrystalline, ceramics, glass-ceramics etc. have been well explained by Johnscher's power law given by A. K. Jonscher's in 1978 expressed mathematically in following form;

$$
\sigma(\omega, T)=\sigma_{d c}+B \omega^{s}(T)
$$

Where, $B$ is a temperature dependent constant, $s$ is power exponent parameter which usually lies in between 0 to $1, \omega$ is angular frequency $\omega=2 \pi f$, and $f$ is angular frequency. The Jonscher's power law has been further modified as [46];

$$
\sigma(f, T)=\sigma_{d c}\left[1+\left(\frac{\mathrm{f}}{\mathrm{f}_{h}}\right)^{s}\right]
$$

In above equation $\mathrm{f}_{h}=\left(\frac{\sigma_{d c}}{B}\right)^{1 / s}$, and the parameter $s$ is an important parameter called as power exponent that usually lies between $s=0$ to 1 such that $s=0$ indicates frequency independent region (linear plateau parallel to frequency axis) called as dc conductivity $\left(\sigma_{\mathrm{dc}}\right)$, and $s=1$ indicates frequency dependence of conductivity. Frequency dependence of ac conductivity for all samples were depicted in Fig. 9 (a) for a reference sample B2LS2. Since the frequency dependence of ac conductivity for rest samples are similar to B2LS2 with only change in their magnitude so their plots have been given in supplementary file. The value of $\sigma_{d c}, \mathrm{f}_{h}$ and $s$ were obtained at each temperature by employ fitting of equation (9) to experimental data, shown by solid line in each figure. 

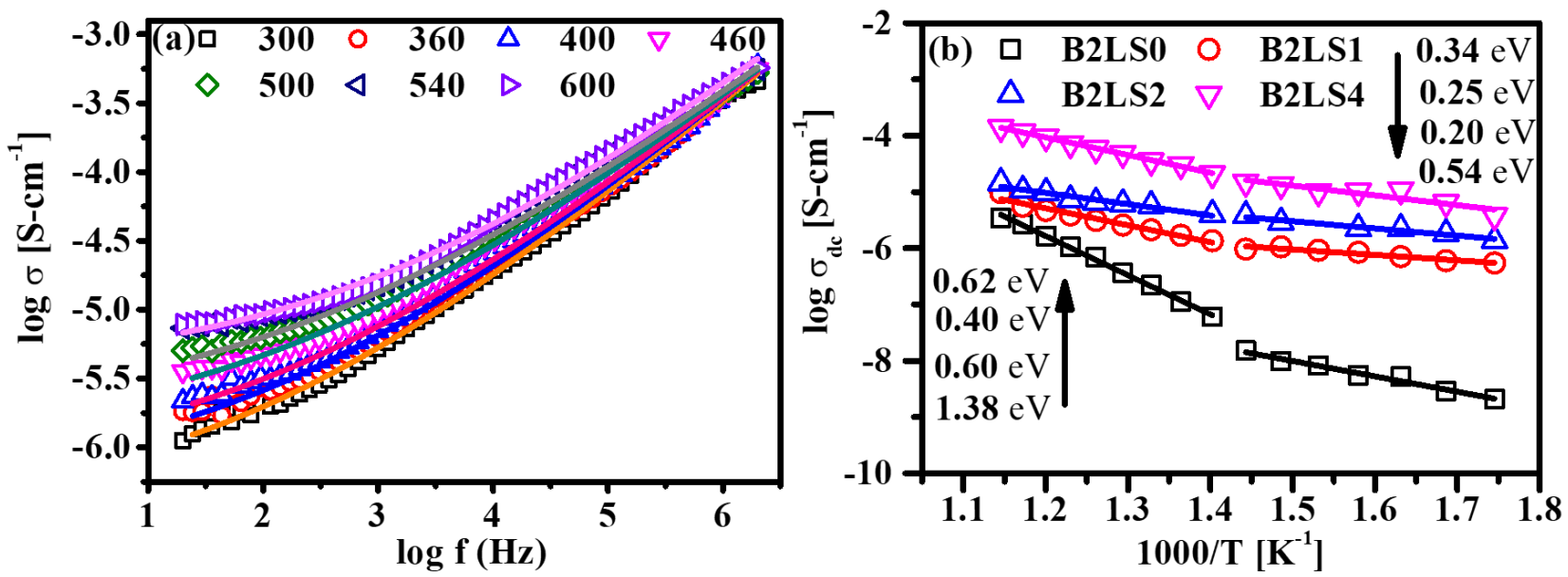

Fig. 9: Variation of ac conductivity as a function of frequency, (a) B2LS2, (b) thermal dependence of dc conductivity for all samples. (Symbols shows the experimental data point and solid line shows fitted data).

The thermal dependence of dc conductivity has been shown in Fig. 9 (b), to study about the conduction mechanism operative in samples. Arrhenius model gives a relation between the dc conductivity and temperature by following relation [7];

$\log \sigma_{d c}=\log \sigma_{o}+\left(-\frac{E_{\text {cond. }}}{k_{B} T}\right)$

Where, $\sigma_{o}$ is preexponential factor, $k_{B}$ is Boltzmann constant and $E_{\text {cond. }}$ is activation energy for the migration of charge species within the samples. The activation energy of each samples has obtained by fitting of equation (10) to experimental data as shown by solid line. The thermal dependence dc conductivity curve shows two regions with different value of activation energy as mentioned in each fig. The conduction mechanism of undoped sample was already reported in literature, therefore, here only it has been considered as reference [15]. The activation energy for undoped samples found to be $1.38 \mathrm{eV}$ (high-temperature region) and $0.54 \mathrm{eV}$ (low temperature region), and with incorporation of La, the activation energy in both regions were decreased, while dc conductivity was increased. This variation can be understood in terms of charge compensation mechanism given by equation (5). Since the doping of La at Ba-site leads to an excess positive charge $\left(2 L a_{B a}{ }^{\circ}\right)$ that can be compensated either by creation of electron $\left(e^{\prime}\right)$ or $V_{B a}^{\prime \prime}$. The dc conductivity of doped sample increases with doping concentration of La suggest that the excess of positive charge on lattice compensated by generation of electron to maintain the overall charge neutrality. Further, the detailed conduction mechanism has been explored in further section. 
In order to understand about the relaxation mechanism in depth, the logarithmic of hopping frequency $\left(f_{h}\right)$ has been plotted with respect to inverse of temperature and shown in Fig. 10. The activation energy for relaxation process $\left(E_{\text {relax. }}\right)$ has been calculated using similar equation to Arrhenius equation that is given by [48];

$\log f_{h}=\log f_{o}+\left(-\frac{E_{\text {relax. }}}{k T}\right)$

Where, $f_{o}$ is preexponential factor, and $k$ is Boltzmann constant. The linear fitting of equation (11) to the experimental data shown by solid line gives the value of $E_{\text {relax. }}$ The value of activation energy found highest for undoped and it decreased with incorporation of La similar to de conductivity.

Since the optical band gap is lowest for sample B2LS1, i.e., $3.23 \mathrm{eV}$, while the activation energy for all samples were found to be less than the separation between valence band and conduction band, that exclude any type of intrinsic conduction. As the samples were synthesized at high temperature, the presence of oxygen vacancy can't be ruled out and given by equation (6). The electrons present in equation (6) may further reduce the valence states of Sn such as; $\mathrm{Sn}^{4+}+2 e^{\prime} \rightarrow \mathrm{Sn}^{2+}$. There are following two ways by which the conduction takes place within samples; (i) either by jumping of electron between the degenerate sites of Sn, (ii) Orientation of electron through dipole $\left(\ddot{V}_{o}-S n^{4+}{ }_{S n^{2+}}{ }^{\prime \prime}\right)$. Based on available literature, the activation energy found greater or equal to $1 \mathrm{eV}$ assigned to the migration of oxygen vacancy while the activation energy lies in between $0.5 \mathrm{eV}$ to $1 \mathrm{eV}$ assigned to the migration of mixed ionic and electronic (i.e., polaronic) and less than $0.5 \mathrm{eV}$ assigned to the purely electronic conduction [49]. The activation energy obtained from dc conductivity is found to be $1.38 \mathrm{eV}$, $0.60 \mathrm{eV}, 0.40 \mathrm{eV}, 0.62 \mathrm{eV}$ in region-1 (High temperature region) and $0.54 \mathrm{eV}, 0.20 \mathrm{eV}, 0.25$ $\mathrm{eV}, 0.34 \mathrm{eV}$ in region-2 (Low temperature region) for samples B2LS0, B2LS1, B2LS2 and B2LS4 respectively. However, activation energy obtained from hopping frequency is found to be $1.61 \mathrm{eV}, 0.52 \mathrm{eV}, 0.70 \mathrm{eV}, 0.82 \mathrm{eV}$ in region-1 (High temperature region) and $0.51 \mathrm{eV}, 0.35$ $\mathrm{eV}, 0.31 \mathrm{eV}, 0.44 \mathrm{eV}$ in region-2 (Low temperature region) for samples B2LS0, B2LS1, B2LS2 and B2LS4 respectively. The activation energy derived from dc conductivity and hopping frequency are nearly identical, implying that the origin of conduction and relaxation mechanisms in both processes are the same. The higher value of activation energy is observed for undoped sample may indicates a strong association among the defect dipoles $\left(\ddot{V}_{o}-\right.$ $\mathrm{Sn}^{4+} \mathrm{Sn}^{2+}{ }^{\prime \prime}$ ), that need small amount of energy initially for dissociation and then it migrates. 
The dc conductivity results in high temperature region reflect the ionic migration that transformed to polaronic with incorporation of $\mathrm{La}$, while the low temperature region reflects the electronic conduction that remain same for all samples. Since, the conductivity in undoped samples results due to migration of oxygen vacancy and electron, however, the activation energy gradually decreased with incorporation of La that observed either of decrease in oxygen vacancy or increase of hopping length. The dc conductivity was found to be increased that may be due to either larger charge carrier concentration or higher mobility. Increasing in mobility may also leads to an increase in activation energy that does not support the present result. Therefore, the reason for increasing the dc conductivity is increase of charge carrier concentration. Also, La acts as donor for Ba-site of $\mathrm{Ba}_{2} \mathrm{SnO}_{4}$ and the overall charge has been compensated by creation of electron as given by equation (5). Due to electronic charge compensation mechanism, the number of electrons within the samples also increase and it results to rise in dc conductivity.

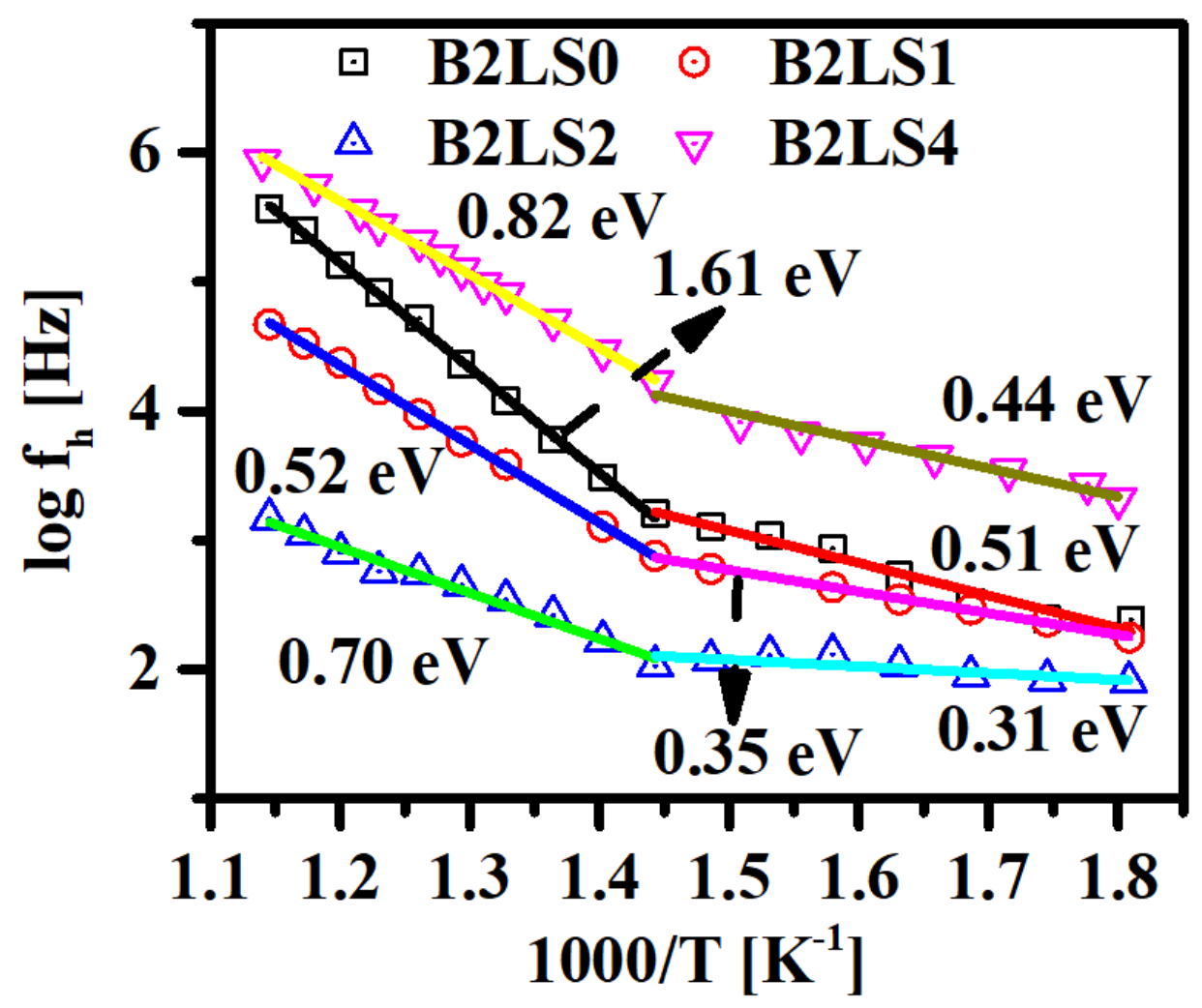

Fig. 10: Thermal dependence of hopping frequency $\left(f_{h}\right)$ for all samples. Symbols show the experimental data point and the solid line represent the fitting of equation (11). 
In order to see the role of temperature and composition on conduction and relaxation process occurred in sample; the time temperature superposition principle (TTSP) has been taken into account and given by [50]:

$\frac{\sigma}{\sigma_{d c}}=F\left(\frac{f}{f_{h}}\right)$

The TTSP curve has been generated by scaling the frequency axis $(f)$ with hopping frequency $\left(f_{h}\right)$ and ac conductivity $(\sigma)$ with dc conductivity $\left(\sigma_{d c}\right)$ at each temperature and shown for sample B2LS1 and B2LS4 in Fig. 11 (a) and (b) respectively. All the thermal curve perfectly superimposes on each other at all temperatures, indicating reflects that the sources of charge carriers are remain same at all temperature. Therefore, the conduction in sample within low temperature region take place via hopping of electron between the degenerate sites of Sn while in high temperature region the conduction take place via orientation of electron from $\mathrm{Sn}^{4+}$ to $\mathrm{Sn}^{2+}$ through polaron.
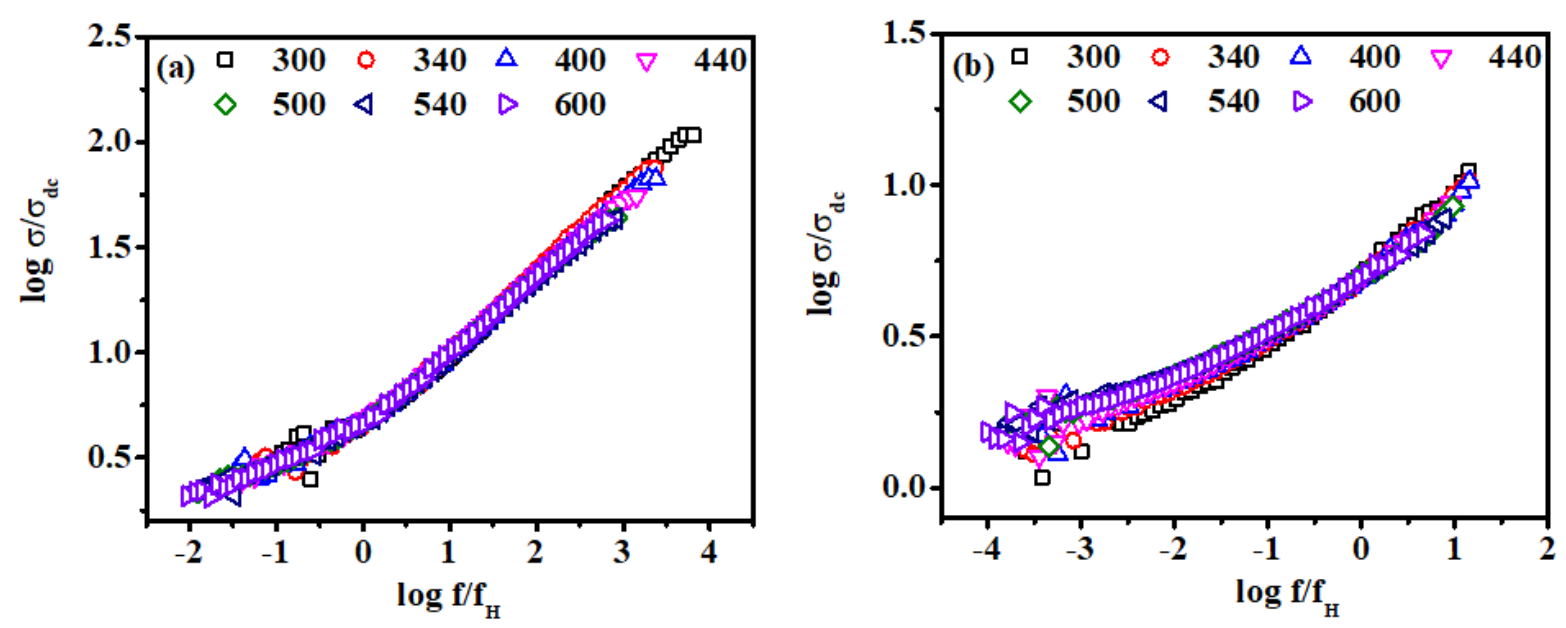

Fig. 11: Time-Temperature Superposition Principle (TTSP) curve for samples, (a) B2LS1 (b) B2LS4.

In order to explore the conduction mechanism, the variation of power exponent as a function of temperature has been illustrated in Fig. 12. The power exponent represents the dimensionality of conduction as well as the strength among the host lattice with the mobile species. Based on the variation of power exponent, different models are well discussed in the literature to explore the ac conduction mechanism involved in the samples [15]. Few of them are given below; 
1. Minimum values of $s$ followed by an increase suggests that the conduction in samples occurred by overlapping of large polaron tunneling (OLPT) model.

2. Temperature independent variation of $s$ suggests the occurrence of conduction through Quantum mechanical tunneling (QMT).

3. Decrease of $s$ with temperature suggests the presence of correlated barrier hopping $(\mathrm{CBH})$ in the samples.

Based on the variation observed for power exponent, the OLPT model is most suitable to analyse the detailed ac conduction mechanism in present samples.
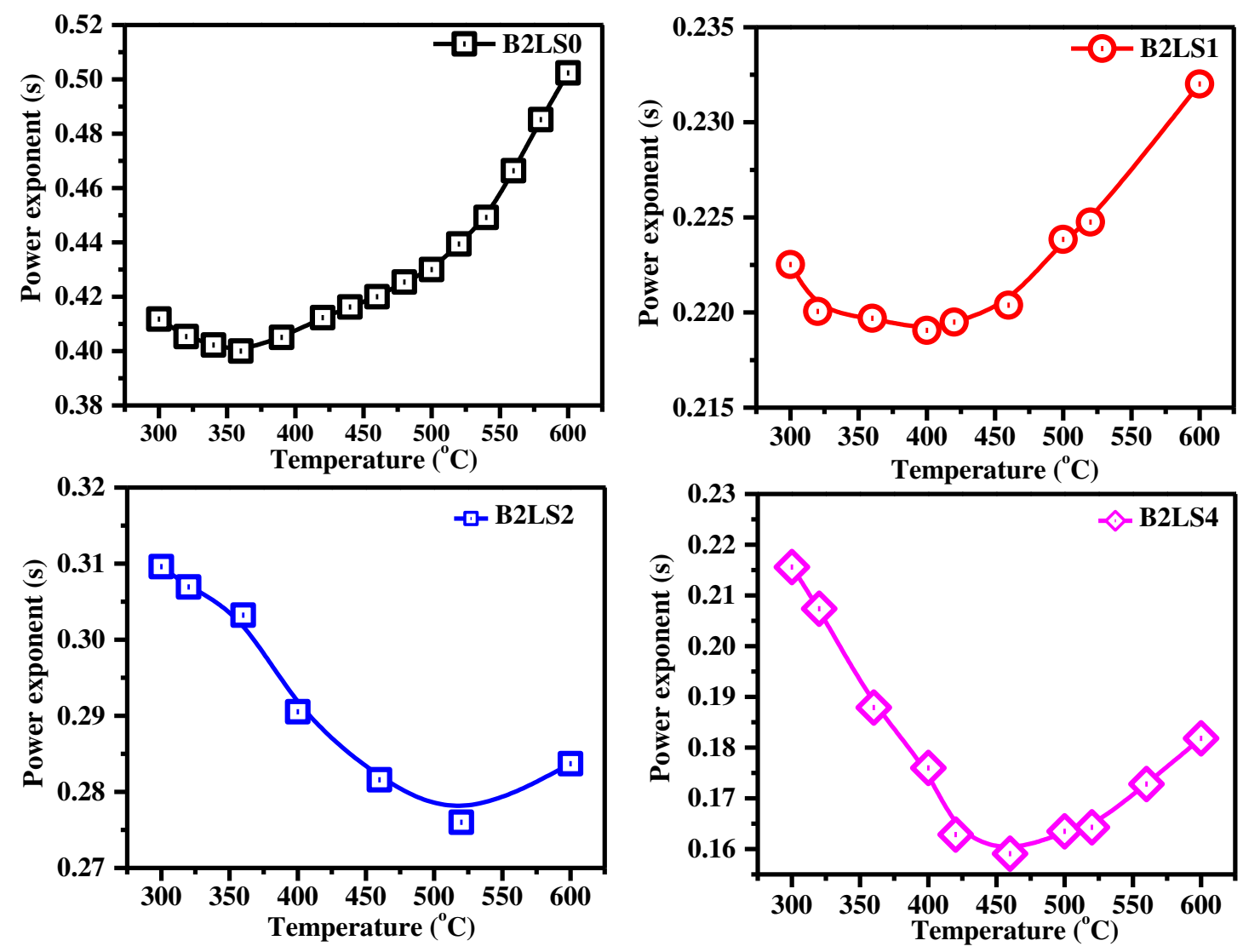

Fig. 12: Variation of power exponent as a function of temperature for all samples.

In OLPT model, the power exponent $s$ depend both on frequency and temperature. Initially $s$ had dropped to a minimum value with increasing temperature and then escalated with further increase in temperature [51]. The ac conductivity in OLPT model is usually given by following equation [52];

$\sigma_{a c}(\omega)=\left[\frac{\pi^{4} e^{2} k_{B}{ }^{2} T^{2} N^{2}}{12}\right] * \frac{\omega R_{\omega}{ }^{4}}{2 \alpha k_{B} T+\frac{W_{H O} O_{p}}{R_{\omega}{ }^{2}}}$ 
Here, $\mathrm{k}_{\mathrm{B}}$ is the Boltzmann's constant, $N$ is the number of defect state near the Fermi level, $\alpha$ is the inverse of localization length, $R_{\omega}$ is the hopping length, $W_{H O}$ is the energy required for overlapping of the polaron and $r_{p}$ is the length of polaron. The hopping length is further determined by solving the quadratic equation [53];

$R^{\prime}{ }_{\omega}^{2}+\left[W_{H O} \beta+\ln \left(\omega \tau_{o}\right)\right] R_{\omega}^{\prime}-W_{H O} r_{p}^{\prime} \beta=0$

Where $\beta=1 / k_{B} T, R_{\omega}^{\prime}=2 \alpha R_{\omega}$, and $r_{p}^{\prime}=2 \alpha r_{p}$. Also, the frequency exponent $s$ can be expressed in this model is given by;

$S=1-\frac{8 \alpha R_{\omega}+\frac{6 W_{H O} r_{p}}{R_{\omega} k_{B} T}}{\left[2 \alpha R_{\omega}+\frac{W_{H O} O_{p}}{R_{\omega} k_{B} T}\right]^{2}}$

Fig. 13 shows the temperature dependence of AC conductivity at constant frequency $10 \mathrm{KHz}$ for all samples. The experimental data were fitted to the equations (12) using $N\left(E_{f}\right), W_{H O}, r_{p}$, $R_{\omega}$, and $\alpha$ as the initial parameters [52]. All the parameters were derived from the least square fitting and given in Table 3.

Table 3: Parameters obtained from OLPT model fitting.

\begin{tabular}{|l|l|l|l|l|l|}
\hline $\begin{array}{l}\text { Sample } \\
\text { Code }\end{array}$ & $\begin{array}{l}\boldsymbol{N}\left(\boldsymbol{E}_{\boldsymbol{f}}\right) \quad\left(\mathbf{x} \mathbf{1 0}^{\mathbf{1 9}}\right) \\
\left(\mathbf{e v}^{-\mathbf{1}} \cdot \mathbf{c m}^{-\mathbf{3}}\right)\end{array}$ & $\boldsymbol{\alpha}\left(\AA^{-\mathbf{1}}\right)$ & $\boldsymbol{r}_{\boldsymbol{p}}(\AA)$ & $\boldsymbol{R}_{\boldsymbol{\omega}}(\AA)$ & $\boldsymbol{W}_{\text {но }}(\mathbf{e V})$ \\
\hline B2LS0 & 1.44 & 0.220 & 0.836 & 3.62 & 1.06 \\
\hline B2LS1 & 2.06 & 0.219 & 0.940 & 3.59 & 0.86 \\
\hline B2LS2 & 3.14 & 0.192 & 1.092 & 3.53 & 0.65 \\
\hline B2LS4 & 4.56 & 0.195 & 1.061 & 3.27 & 0.43 \\
\hline
\end{tabular}




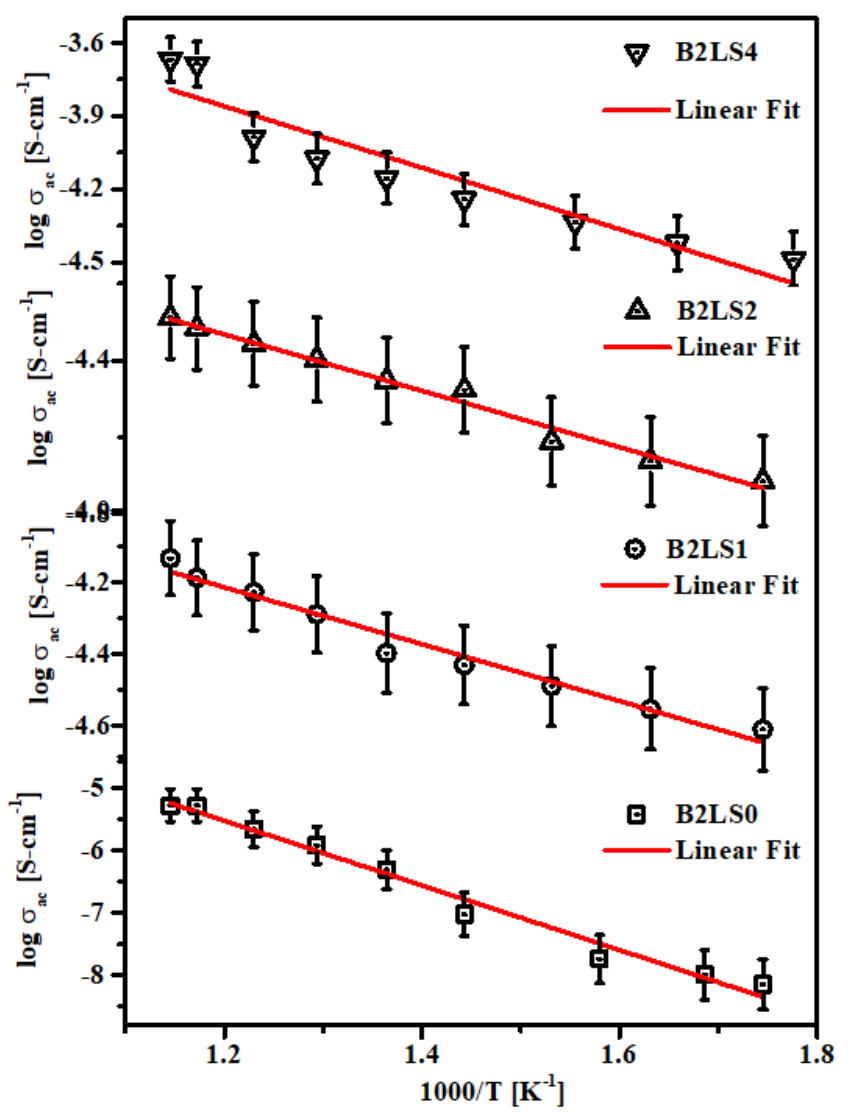

Fig. 13: Variation of power exponent $(s)$ with respect to inverse of temperature. Symbols shows experimental data and solid line shows fitted data using equation (13).

From Table 3, it has been noticed that with incorporation of $\mathrm{La}^{3+}$ the value of charge carrier concentration increases that might be possible reason for rise in dc conductivity. It is also clear that the value of hopping length $\left(R_{\omega}\right)$ decreases with $\mathrm{La}^{3+}$, that follow similar trend of the inter atomic distance given in Table 2 such as Ba-O1 = 2.89019- $2.8544(\AA), \mathrm{Ba}-\mathrm{O} 2=2.9354-2.9303$ $(\AA), \mathrm{Sn}-\mathrm{O} 1=2.06285-2.0729(\AA)$, and Sn-O2 = 1.8122-2.2605 (§). Also, it has been noticed that the interatomic distance is found lower than the hopping length that suggests the formation of large polaron. So, the conduction in the sample takes place via formation of the overlapping of large polaron. In case of large polarons, the spatial extent of the polaron is larger compared to the interatomic distance [54]. Since the $W_{H O}$ is the value of activation energy for the hopping of charge carrier between neighbouring sites via the overlapping. The value of $W_{H O}$ obtained for present sample is nearly equal to the activation energy obtained from dc conductivity that further supports the migration of charge carrier between the degenerate sites through overlapping of polaron. Further, the value of polaron radius increases with doping concentration of $\mathrm{La}^{3+}$ that need lower amount of energy for the migration of polaron. 
As previously mentioned, $\mathrm{Ba}_{2} \mathrm{SnO}_{4}$ is made up of a perovskite $\mathrm{BaSnO}_{3}$ and a $\mathrm{BaO}$ sheet, so the result of $\mathrm{Ba}_{2} \mathrm{SnO}_{4}$ can be compared to that of $\mathrm{BaSnO}_{3} . \mathrm{BaSnO}_{3}$ is crystallized into cubic crystal structure with $\mathrm{Ba} 2+$ ions in dodecahedral site and $\mathrm{Sn}^{4+}$ ion is at octahedral site. The distance between the Sn-O and Ba-O were 2.05779 ̊, $2.91015 \AA$ respectively [46]. Also, a comparison between the dc conductivity of La-doped $\mathrm{BaSnO}_{3}$ and with present investigated samples at $600^{\circ} \mathrm{C}$ has been made [29]. The $\mathrm{BaSnO}_{3}$ doped with $\mathrm{La}(1 \%)$ had dc conductivity of $1.41 \times 10^{-}$ ${ }^{4} \mathrm{~S}-\mathrm{cm}^{-1}$ while B2LS1 had a dc conductivity of $1.26 \times 10^{-5} \mathrm{~S}_{-\mathrm{cm}^{-1}}$. Since the conduction in $\mathrm{BaSnO}_{3}$ is governed by the nearest neighbour site either O-Sn-O or Sn-O-Sn which is remain same in $\mathrm{Ba}_{2} \mathrm{SnO}_{4}$. Conduction occurs in samples between two lattice sites with lower potential energy through the defect only when the defect relaxes before the two minima of lattice potential energy coincide with the lattice site [55]. Furthermore, the presence of a Ba-O layer exerted pressure on the perovskite $\mathrm{BaSnO}_{3}$, causing a difference in the crystal structure of $\mathrm{BaSnO}_{3}$ found in $\mathrm{Ba}_{2} \mathrm{SnO}_{4}$ and regular $\mathrm{BaSnO}_{3}$. Furthermore, the interatomic distance of our compounds Sn-O1, Sn-O2 is found to be greater than the interatomic distance of $\mathrm{Sn}-\mathrm{O}$ in $\mathrm{BaSnO}_{3}$. As a result of this explanation, we can infer that there is a difference in conductivity between these two compounds. Thus, in the present sample, conductivity is ensured by the overlapping of large polarons within the large tunnels, while in $\mathrm{BaSnO}_{3}$, it is ensured by the overlapping of small polarons within the small tunnels [56].

Moreover, the contribution of grain, grain boundary, and electrode to the overall electrical properties can be studied using Nyquist plot. The Nyquist plot has been shown in Fig. 14 for all samples obtained at selected temperatures such as $300^{\circ} \mathrm{C}, 400^{\circ} \mathrm{C}, 500^{\circ} \mathrm{C}$, and $600^{\circ} \mathrm{C}$. A depressed semi-circular arc has been observed for all samples. Further, the Nyquist plot has been fitted with a Cole-Cole equation to determine the contribution of grain and grain boundary is given by [57];

$$
\left(Z^{\prime}-\frac{R}{2}\right)^{2}+Z^{\prime \prime}=\left(\frac{R}{2}\right)^{2}
$$

The resistance of grain and grain boundary were determined by employing the equation (16) to experimental data shown by solid line in Fig. 14. Ideally, three semi-circular arcs have been observed that assigned to the grain, grain boundary, and electrode contribution respectively [51]. Although they are found to be present separately not only at higher frequency but also due to large difference in their relaxation time. The measurement cannot be performed at higher frequency due to limitation of instrument used in present investigation. In present sample, only a singular arc has been observed that indicates the resistance can be given by $R_{\text {total }}=$ 
$\mathrm{R}_{\mathrm{g}}+\mathrm{R}_{\mathrm{gb}}+\mathrm{R}_{\mathrm{elec}}$. Since the arcs observed in present investigation are depressed that reflects the distribution of relaxation time among the grains/grain boundaries. Further, the SEM analysis shows agglomeration among the grains that might be one of the reasons that the contribution of grain boundaries was not separable. The value of resistance obtained by fitting of cole-cole equation has been given in Table 4. At the top of the semicircle, following condition should be satisfied [57];

$2 \pi f_{\max } R C=1$

The value of effective capacitance was calculated using the frequency and resistance at each temperature for all samples and given in Table 4. The value of effective capacitance calculated from equation (17) was found to be in agreement with the effective capacitance arised due to major effect of interface present within the samples [22,58]. Therefore, in order to further study about the role of grain to the electrical conduction and relaxation mechanism, the thermal dependence of effective resistance was taking into account.

Table 4: The value of Resistance, capacitance, frequency $\left(f_{\max }\right)$ obtained at different temperatures for all samples.

\begin{tabular}{|c|c|c|c|c|}
\hline Sample & Temperature $\left({ }^{\circ} \mathrm{C}\right)$ & $\mathrm{R}_{\text {total }}(\mathrm{ohm})$ & $\mathrm{f}_{\max }(\mathrm{Hz})$ & $\mathrm{C}_{\text {total }}(\mathrm{F})$ \\
\hline \multirow{4}{*}{ B2LS0 } & 300 & $8.69 \times 10^{6}$ & 253 & $4.54 \times 10^{-10}$ \\
\hline & 400 & $3.75 \times 10^{6}$ & 552 & $4.83 \times 10^{-10}$ \\
\hline & 500 & $2.98 \times 10^{6}$ & 671 & $5.00 \times 10^{-10}$ \\
\hline & 600 & $2.14 \times 10^{6}$ & 991 & $4.72 \times 10^{-10}$ \\
\hline \multirow{4}{*}{ B2LS1 } & 300 & $4.70 \times 10^{6}$ & 307 & $6.93 \times 10^{-10}$ \\
\hline & 400 & $2.93 \times 10^{6}$ & 671 & $5.08 \times 10^{-10}$ \\
\hline & 500 & $1.24 \times 10^{6}$ & 990 & $8.14 \times 10^{-10}$ \\
\hline & 600 & $7.84 \times 10^{5}$ & 1463 & $8.72 \times 10^{-10}$ \\
\hline \multirow{4}{*}{ B2LS2 } & 300 & $3.32 \times 10^{6}$ & 453 & $6.64 \times 10^{-10}$ \\
\hline & 400 & $1.97 \times 10^{6}$ & 815 & $6.23 \times 10^{-10}$ \\
\hline & 500 & $1.14 \times 10^{6}$ & 1204 & $7.29 \times 10^{-10}$ \\
\hline & 600 & $7.38 \times 10^{5}$ & 1779 & $7.62 \times 10^{-10}$ \\
\hline \multirow{4}{*}{ B2LS4 } & 300 & $3.87 \times 10^{5}$ & 670 & $3.87 \times 10^{-9}$ \\
\hline & 400 & $2.22 \times 10^{5}$ & 1463 & $3.08 \times 10^{-9}$ \\
\hline & 500 & $1.34 \times 10^{5}$ & 2162 & $3.45 \times 10^{-9}$ \\
\hline & 600 & $8.60 \times 10^{4}$ & 3194 & $3.64 \times 10^{-9}$ \\
\hline
\end{tabular}


The conduction mechanism of charge carrier within grain has been discussed by determining the grain resistance for all samples.
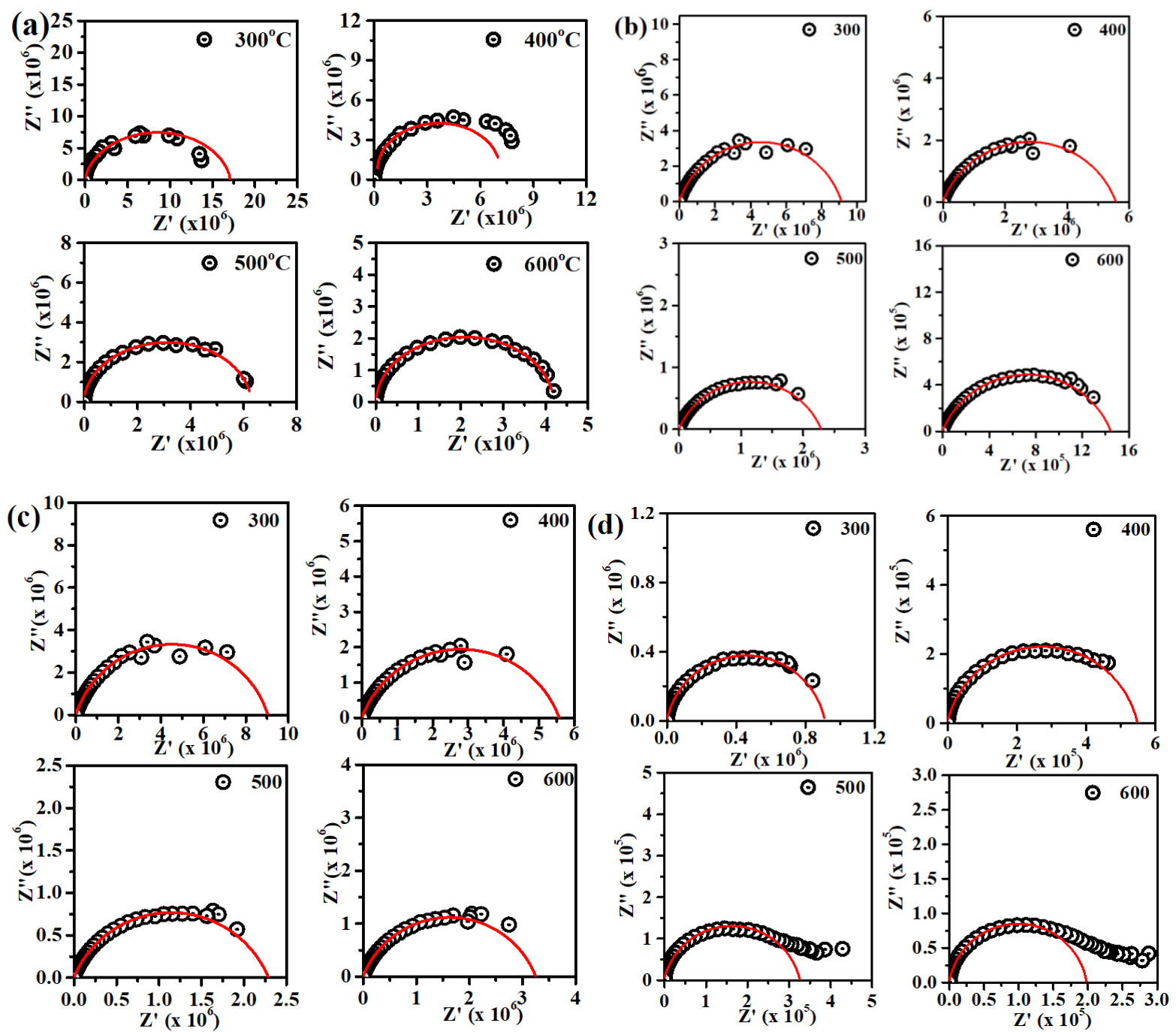

Fig. 14: Nyquist Plot obtained for all samples (a) B2LS0, (b) B2LS1, (c) B2LS2, (d) B2LS4, symbols represent the experimental data point, and solid line shows fitting using equation (16). The illustration of logarithmic of resistance derived from the cole-cole fitting with respect to inverse of temperature was shown in Fig. 15. The activation energy required for the migration of charge carrier within the sample through grain/grain boundary/electrode has been calculated by employing fitting of Arrhenius equation to the experimental data and given in the inset of each fig. The value of activation energy obtained for grain in present case assigned to the migration of electrons between the degenerate sites of $\mathrm{Sn}^{4+}$ and $\mathrm{Sn}^{2+}$. Since the order of capacitance reflects the contribution of interface, that might be due to presence of multivalent ion at the interface such as $\mathrm{Sn}^{4+}$. The multivalent ion captures the electron present at the interface and reduced the multivalent states of $\mathrm{Sn}^{4+}$ to $\mathrm{Sn}^{2+}$. Since the grain boundary shows higher insulating than grain so that it needs slightly higher activation energy for the migration 
of charge carrier [15,59]. With increasing the doping concentration, the value of activation energy found to be gradual increase that might be due to large size of polaron (See Table 3 ).
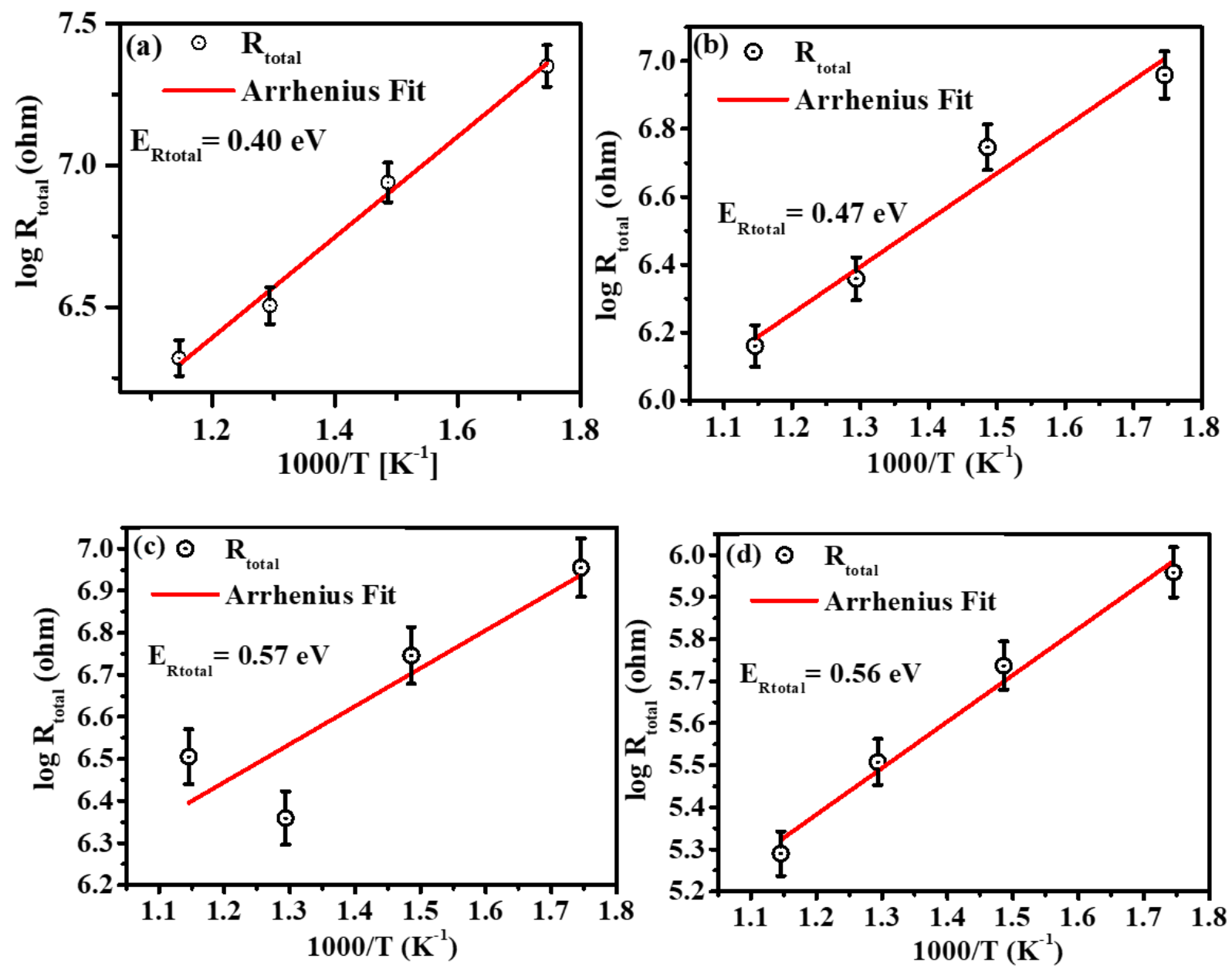

Fig. 15: Variation between the logarithmic of total resistance $\left(\mathrm{R}_{\text {total }}\right)$ with inverse of temperature for all samples, (a) B2LS0 (b) B2LS1 (c) B2LS2 (d) B2LS4

Based on the value of activation energy, the present materials can be utilized as mixed ionic and electronic conductor and semiconductor device applications.

\section{Conclusions:}

A few compositions of the system $\mathrm{Ba}_{2-\mathrm{x}} \mathrm{La}_{x} \mathrm{SnO}_{4}$ (where $\mathrm{x}=0,0.01,0.02,0.04,0.06,0.10$ ) have been successfully synthesized using the solid-state ceramic route. The XRD studies along with FTIR, Raman and UV-Vis. study suggests the solubility of La at the site of $\mathrm{Ba}$ in $\mathrm{Ba}_{2} \mathrm{SnO}_{4}$ was limited between 4 to 6 atoms \%. The compositions having a doping concentration above than 4 atom \% contains impurity of $\mathrm{La}_{2} \mathrm{Sn}_{2} \mathrm{O}_{7}$ pyrochlore phase. Rietveld refinement studies performed on XRD data indicated lower unit cell volume, density, and bond length $\mathrm{Ba} / \mathrm{La}-\mathrm{O} 1$ and $\mathrm{Ba} / \mathrm{La}-\mathrm{O} 2$. The incorpration of La results decrease of crystallite size, and average grain 
size whereas increase of micro-strain due to lower ionic radii of $\mathrm{La}^{3+}$ than $\mathrm{Ba}^{2+}$. The optical band gap of all samples obtained using Tauc plot was found to be in the range of (3.23-3.75) $\mathrm{eV}$ that indicates semiconducting nature of sample. The ac conductivity spectra of all samples follow universal Johnscher's power law. The value of $\sigma_{d c}$ and $f_{h}$ derived using Johnscher's power law indicating the presence of Arrhenius type conduction in samples. Two regions of conduction found within the investigated temperature region. The values of activation energy obtained from $\sigma_{d c}$ and $f_{h}$ was found almost same that indicates the similar sources are responsible for dc and ac conduction. The ac conduction occurs within the sample via the formation of large polaron overlapping between the neighbouring site that also support from the bond length $\mathrm{Ba} / \mathrm{La}-\mathrm{O} 1, \mathrm{Ba} / \mathrm{La}-\mathrm{O} 2, \mathrm{Sn}-\mathrm{O} 1, \mathrm{Sn}-\mathrm{O} 2$ and $R_{\omega}$ derived from OLPT model fitting. Also, the scaled conductivity with respect to scaled frequency nicely superimposed on each other, suggesting that the source of charge carrier found to be same at all temperatures. The major contribution of grain was observed from Nyquist plot further suggesting that the conduction takes place via the overlapping of large polaron tunnelling. Based on the studies, the present materials could be utilized as semiconductor and mixed ionic and electronic conductor (MIECs) in fuel cell application.

\section{Acknowledgement:}

The authors are grateful to the Head Department of Physics, Head Department of Pharmaceutics, Head Department of Metallurgical Engineering and Coordinator, Central Instrument Facility Centre (CIFC), IIT(BHU), Varanasi for providing the experimental facilities required for the characterization of the synthesized samples.

\section{Conflict of Interest}

There is no any conflict of interest in this manuscript.

\section{Data availability statement}

The raw/processed data required to reproduce these findings cannot be shared at this time as the data also a part of an ongoing study.

\section{References:}

[1] D. Lee, H. Lee, Controlling Oxygen Mobility in Ruddlesden-Popper Oxides, Materials (Basel). 10 (2017) 368. https://doi.org/10.3390/ma10040368.

[2] C. Navas, H.C. Zur Loye, Conductivity studies on oxygen-deficient Ruddlesden- 
Popper phases, Solid State Ionics. 93 (1996) 171-176. https://doi.org/10.1016/S01672738(96)00515-2.

[3] P. V. Balachandran, D. Puggioni, J.M. Rondinelli, Crystal-Chemistry Guidelines for Noncentrosymmetric A 2 BO 4 Ruddlesden-Popper Oxides, Inorg. Chem. 53 (2014) 336-348. https://doi.org/10.1021/ic402283c.

[4] T.L. Meyer, R. Jacobs, D. Lee, L. Jiang, J.W. Freeland, C. Sohn, T. Egami, D. Morgan, H.N. Lee, Strain control of oxygen kinetics in the Ruddlesden-Popper oxide La1.85Sr0.15CuO4, Nat. Commun. 9 (2018) 92. https://doi.org/10.1038/s41467-01702568-z.

[5] M. Wei, W. Che, H. Li, Z. Wang, F. Yan, Y. Liu, J. Liu, Ruddlesden-Popper type $\mathrm{La} 2 \mathrm{NiO} 4+\delta$ oxide coated by $\mathrm{Ag}$ nanoparticles as an outstanding anion intercalation cathode for hybrid supercapacitors, Appl. Surf. Sci. 484 (2019) 551-559. https://doi.org/10.1016/j.apsusc.2019.04.015.

[6] H. Weng, Y. Kawazoe, X. Wan, J. Dong, Electronic structure and optical properties of layered perovskites Sr2 M O4 (M=Ti, V, Cr, Mn, and Co): An ab initio study, Phys. Rev. B - Condens. Matter Mater. Phys. 74 (2006) 1-9. https://doi.org/10.1103/PhysRevB.74.205112.

[7] U. Kumar, S. Upadhyay, Sr 2 SnO 4 Ruddlesden Popper Oxide: Future Material for Renewable Energy Applications, in: Green Energy, Wiley, 2020: pp. 221-250. https://doi.org/10.1002/9781119760801.ch8.

[8] K.H. Lee, Y.F. Wang, S.W. Kim, H. Ohta, K. Koumoto, Thermoelectric Properties of Ruddlesden?Popper Phase n-Type Semiconducting Oxides: La-, Nd-, and Nb-Doped Sr 3 Ti 2 O 7, Int. J. Appl. Ceram. Technol. 4 (2007) 326-331. https://doi.org/10.1111/j.1744-7402.2007.02147.x.

[9] R. Zhang, M.S. Senn, M.A. Hayward, Directed Lifting of Inversion Symmetry in Ruddlesden-Popper Oxide-Fluorides: Toward Ferroelectric and Multiferroic Behavior, Chem. Mater. 28 (2016) 8399-8406. https://doi.org/10.1021/acs.chemmater.6b03931.

[10] L. Miao, J. Hou, Z. Gong, Z. Jin, W. Liu, A high-performance cobalt-free RuddlesdenPopper phase cathode $\mathrm{La} 1 \cdot 2 \mathrm{Sr} 0 \cdot 8 \mathrm{Ni} 0 \cdot 6 \mathrm{Fe} 0 \cdot 4 \mathrm{O} 4+\delta$ for low temperature protonconducting solid oxide fuel cells, Int. J. Hydrogen Energy. 44 (2019) 7531-7537. https://doi.org/10.1016/j.ijhydene.2019.01.255.

[11] G. Amow, S.J. Skinner, Recent developments in Ruddlesden-Popper nickelate systems for solid oxide fuel cell cathodes, J. Solid State Electrochem. 10 (2006) 538-546. 
https://doi.org/10.1007/s10008-006-0127-X.

[12] A. Jun, T. Lim, J. Shin, G. Kim, Electrochemical properties of B-site Ni doped layered perovskite cathodes for IT-SOFCs, Int. J. Hydrogen Energy. 39 (2014) 20791-20798. https://doi.org/10.1016/j.ijhydene.2014.06.136.

[13] A. Manthiram, J.-H. Kim, Y.N. Kim, K.-T. Lee, Crystal chemistry and properties of mixed ionic-electronic conductors, J. Electroceramics. 27 (2011) 93-107. https://doi.org/10.1007/s10832-011-9635-X.

[14] K.S. Yun, J.H. Park, Y. Kwon, D.Y. Kim, C.-Y. Yoo, J.H. Yu, J.H. Joo, A new strategy for enhancing the thermo-mechanical and chemical stability of dual-phase mixed ionic electronic conductor oxygen membranes, J. Mater. Chem. A. 4 (2016) 13549-13554. https://doi.org/10.1039/C6TA04361E.

[15] U. Kumar, S. Upadhyay, Structural, Optical and Electrical Properties of Ruddlesden Popper Oxide Ba2SnO4, J. Electron. Mater. 48 (2019) 5279-5293. https://doi.org/10.1007/s11664-019-07336-X.

[16] A.P. Khandale, J.D. Punde, S.S. Bhoga, Electrochemical performance of strontiumdoped neodymium nickelate mixed ionic-electronic conductor for intermediate temperature solid oxide fuel cells, J. Solid State Electrochem. 17 (2013) 617-626. https://doi.org/10.1007/s10008-012-1892-3.

[17] C. Jin, J. Liu, Y. Zhang, J. Sui, W. Guo, Characterization and electrochemical performances of $\mathrm{Ba} 2-\mathrm{xSrxFeO} 4+\delta$ as a novel cathode material for intermediatetemperature solid oxide fuel cells, J. Power Sources. 182 (2008) 482-488. https://doi.org/10.1016/j.jpowsour.2008.04.044.

[18] S. Science, Sol-gel (combustion) synthesis and characterization of different alkaline earth metal (Ca, Sr, Ba) stannates, J. Sol-Gel Sci. Technol. 64 (2012) 643-652. https://doi.org/10.1007/s10971-012-2896-2.

[19] A. Kareiva, S. Šakirzanovas, T. Jüstel, M. Van Bael, A. Katelnikovas, D. Dutczak, D. Enseling, A. Stanulis, A. Hardy, Luminescence properties of Sm3+-doped alkaline earth ortho-stannates, Opt. Mater. (Amst). 36 (2014) 1146-1152. https://doi.org/10.1016/j.optmat.2014.02.018.

[20] H. Gao, Y. Wang, Photoluminescence of Eu3+ activated Ba2SnO4 under ultravioletvacuum ultraviolet excitation, J. Mater. Res. 21 (2006) 1857-1861. https://doi.org/10.1557/jmr.2006.0230.

[21] J. Zhang, R. Hu, Q. Qin, D. Wang, B. Liu, Y. Wen, M. Zhou, Y. Wang, The origin of two quenching concentrations and unusual afterglow behaviors of $\mathrm{Ba} 2 \mathrm{SnO} 4: \mathrm{Sm} 3+$ 
phosphor, J. Lumin. 132 (2012) 2590-2594.

https://doi.org/10.1016/j.jlumin.2012.05.027.

[22] U. Kumar, K. Ankur, D. Yadav, S. Upadhyay, Synthesis and characterization of Ruddlesden-Popper system (Ba1-xSrx)2SnO4, Mater. Charact. 162 (2020) 110198. https://doi.org/10.1016/j.matchar.2020.110198.

[23] M. Yasukawa, T. Kono, K. Ueda, H. Yanagi, H. Hosono, High-temperature thermoelectric properties of La-doped BaSnO3 ceramics, Mater. Sci. Eng. B. 173 (2010) 29-32.

[24] B.C. Luo, J. Zhang, J. Wang, P.X. Ran, Structural, electrical and optical properties of lanthanum-doped barium stannate, Ceram. Int. 41 (2015) 2668-2672.

[25] C. Shan, T. Huang, J. Zhang, M. Han, Y. Li, Z. Hu, J. Chu, Optical and Electrical Properties of Sol-Gel Derived Ba1-x La x SnO3 Transparent Conducting Films for Potential Optoelectronic Applications, J. Phys. Chem. C. 118 (2014) 6994-7001.

[26] U.S. Alaan, F.J. Wong, J.J. Ditto, A.W. Robertson, E. Lindgren, A. Prakash, G. Haugstad, P. Shafer, D. Johnson, E. Arenholz, Magnetism and transport in transparent high-mobility BaSnO 3 films doped with La, Pr, Nd, and Gd, Phys. Rev. Mater. 3 (2019) 124402.

[27] K.K. James, P.S. Krishnaprasad, K. Hasna, M.K. Jayaraj, Structural and optical properties of La-doped BaSnO3 thin films grown by PLD, J. Phys. Chem. Solids. 76 (2015) 64-69. https://doi.org/10.1016/j.jpcs.2014.07.024.

[28] Q. Liu, J. Liu, B. Li, H. Li, G. Zhu, K. Dai, Z. Liu, P. Zhang, J. Dai, Composition dependent metal-semiconductor transition in transparent and conductive La-doped BaSnO3 epitaxial films, Appl. Phys. Lett. 101 (2012) 241901.

[29] M.J. Ansaree, S. Upadhyay, Electrical characterization of porous La-doped BaSnO3 using impedance spectroscopy, Ionics (Kiel). 21 (2015) 2825-2838. https://doi.org/10.1007/s11581-015-1476-1.

[30] M. Yasukawa, T. Kono, K. Ueda, H. Yanagi, H. Hosono, High-temperature thermoelectric properties of La-doped BaSnO3 ceramics, Mater. Sci. Eng. B SolidState Mater. Adv. Technol. 173 (2010) 29-32. https://doi.org/10.1016/j.mseb.2009.10.002.

[31] S. Upadhyay, O. Parkash, D. Kumar, Solubility of lanthanum, nickel and chromium in barium stannate, Mater. Lett. 49 (2001) 251-255. https://doi.org/10.1016/S0167$577 \mathrm{X}(00) 00378-5$.

[32] U. Kumar, D. Yadav, S. Upadhyay, Investigation of structural, optical, and magnetic 
properties of Nd-doped Sr $2 \mathrm{SnO} 4$ Ruddlesden Popper oxide, J. Am. Ceram. Soc. 103 (2020) 5743-5757. https://doi.org/10.1111/jace.17303.

[33] U. Kumar, S. Upadhyay, Structural, microstructure, optical, and dielectric properties of Sr1.99M0.01SnO4 (M: La, Nd, Eu) Ruddlesden-Popper oxide, J. Mater. Sci. Mater. Electron. 31 (2020) 5721-5730. https://doi.org/10.1007/s10854-020-03140-0.

[34] B.J. Kennedy, Neutron powder diffraction study of Sr2SnO4 and Ba2SnO4, Aust. J. Chem. 50 (1997) 917-920. https://doi.org/10.1071/C97117.

[35] T. Truttmann, A. Prakash, J. Yue, T.E. Mates, B. Jalan, Dopant solubility and charge compensation in La-doped SrSnO 3 films, Appl. Phys. Lett. 115 (2019) 152103. https://doi.org/10.1063/1.5119272.

[36] U. Kumar, D. Yadav, A.K. Thakur, K.K. Srivastav, S. Upadhyay, Investigation on phase formation of $\mathrm{Sr} 2 \mathrm{SnO} 4$ and effect of La-doping on its structural and optical properties, J. Therm. Anal. Calorim. 135 (2019) 1987-1999. https://doi.org/10.1007/s10973-018-7432-3.

[37] L. Ai, Z. Wang, C. Cui, W. Liu, L. Wang, Catalytic Oxidation of Soot on a Novel Active Ca-Co Dually-Doped Lanthanum Tin Pyrochlore Oxide, Materials (Basel). 11 (2018) 653. https://doi.org/10.3390/ma11050653.

[38] U. Venkateswaran, K. Strössner, K. Syassen, G. Burns, M.W. Shafer, Pressure dependence of the Raman modes in Sr2TiO4, Solid State Commun. 64 (1987) 12731277. https://doi.org/10.1016/0038-1098(87)90624-7.

[39] A. Kumar, B. Khan, G. Singh, A. Dixit, U. Kumar, M.K. Singh, Structural, microstructure, optical, and electrical properties of Ti-doped CaSnO3prepared by SolGel chemical route, Phys. Scr. 95 (2020) 105807. https://doi.org/10.1088/14024896/abb89f.

[40] U. Kumar, S. Upadhyay, P.A. Alvi, Study of reaction mechanism, structural, optical and oxygen vacancy-controlled luminescence properties of Eu-modified $\mathrm{Sr} 2 \mathrm{SnO} 4$ Ruddlesden popper oxide, Phys. B Condens. Matter. 604 (2021) 412708. https://doi.org/10.1016/j.physb.2020.412708.

[41] U. Kumar, J. Ansaree, S. Upadhyay, Structural and optical characterizations of BaSnO 3 nanopowder synthesized by aqueous sol-gel method, Process. Appl. Ceram. 11 (2017) 177-184.

[42] S. Omeiri, N. Allalou, G. Rekhila, Y. Bessekhouad, M. Trari, Preparation and characterization of nano structured $\mathrm{Ba} 2 \mathrm{SnO} 4$ as a novel photocatalyst material for the chromate reduction, Appl. Nanosci. 4 (2014) 959-966. https://doi.org/10.1007/s13204- 
013-0276-Z.

[43] T. Yamashita, K. Ueda, Blue photoluminescence in Ti-doped alkaline-earth stannates, J. Solid State Chem. 180 (2007) 1410-1413.

https://doi.org/10.1016/j.jssc.2007.02.009.

[44] H. Cheema, S. Kumar, P.A. Alvi, B.L. Choudhary, U. Kumar, Synthesis and physical properties of nanopowder and electrical properties of bulk samples of $\mathrm{ZnFe} 2-\mathrm{xNixO} 4$ (x: 0, 0.05, 0.10), Adv. Powder Technol. 31 (2020) 4241-4252. https://doi.org/10.1016/j.apt.2020.09.001.

[45] H. JAFFE, Piezoelectric Ceramics, J. Am. Ceram. Soc. 41 (1958) 494-498. https://doi.org/10.1111/j.1151-2916.1958.tb12903.x.

[46] U. Kumar, M.J. Ansaree, A.K. Verma, S. Upadhyay, G. Gupta, Oxygen vacancy induced electrical conduction and room temperature ferromagnetism in system BaSn1xNixO3 (0 x 0.20), Mater. Res. Express. 4 (2017) 116304. https://doi.org/10.1088/2053-1591/aa9416.

[47] R.D. Shannon, C.T. Prewitt, Revised values of effective ionic radii, Acta Crystallogr. Sect. B Struct. Crystallogr. Cryst. Chem. 26 (1970) 1046-1048. https://doi.org/10.1107/S0567740870003576.

[48] A. Kumar, B. Khan, V. Yadav, A. Dixit, U. Kumar, M.K. Singh, Rietveld refinement, optical, dielectric and ac conductivity studies of Ba-doped SrSnO3, J. Mater. Sci. Mater. Electron. 31 (2020) 16838-16848. https://doi.org/10.1007/s10854-020-042407.

[49] X. Yang, S. Liu, F. Lu, J. Xu, X. Kuang, Acceptor Doping and Oxygen Vacancy Migration in Layered Perovskite NdBaInO4-Based Mixed Conductors, J. Phys. Chem. C. 120 (2016) 6416-6426. https://doi.org/10.1021/acs.jpcc.6b00700.

[50] S. Kabi, A. Ghosh, Ion dynamics in glassy ionic conductors: Scaling of mean square displacement of mobile ions, EPL (Europhysics Lett. 108 (2014) 36002. https://doi.org/10.1209/0295-5075/108/36002.

[51] N. Chakchouk, B. Louati, K. Guidara, Electrical properties and conduction mechanism study by OLPT model of NaZnPO4 compound, Mater. Res. Bull. 99 (2018) 52-60. https://doi.org/10.1016/j.materresbull.2017.10.046.

[52] A. Zaafouri, M. Megdiche, M. Gargouri, Studies of electric, dielectric, and conduction mechanism by OLPT model of Li4P2O7, Ionics (Kiel). 21 (2015) 1867-1879. https://doi.org/10.1007/s11581-015-1365-7.

[53] S. Nasri, M. Megdiche, M. Gargouri, DC conductivity and study of AC electrical 
conduction mechanisms by non-overlapping small polaron tunneling model in LiFeP 2 O 7 ceramic, Ceram. Int. 42 (2016) 943-951.

https://doi.org/10.1016/j.ceramint.2015.09.023.

[54] S. Singh, P.A. Jha, S. Varma, P. Singh, Large polaron hopping phenomenon in lanthanum doped strontium titanate, J. Alloys Compd. 704 (2017) 707-716. https://doi.org/10.1016/j.jallcom.2017.02.015.

[55] R. Kurre, S. Bajpai, P.K. Bajpai, Synthesis, characterization, optical and transport properties of BaSnO 3 synthesized by wet chemical route, Mater. Sci. Appl. 9 (2018) 92.

[56] S. Murugavel, M. Upadhyay, AC conduction in amorphous semiconductors, J. Indian Inst. Sci. 91 (2011) 303-318.

[57] J.R. Macdonald, W.B. Johnson, Fundamentals of Impedance Spectroscopy, in: Impedance Spectrosc. Theory, Exp. Appl. Second Ed., 2005. https://doi.org/10.1002/0471716243.ch1.

[58] S.K. Kim, M. Miyayama, H. Yanagida, Complex impedance and modulus analysis on electrical anisotropy of layer-structured $\mathrm{BaBi} 4 \mathrm{Ti} 4 \mathrm{O} 15$ single crystal in paraelectric phase, Nippon Seramikkusu Kyokai Gakujutsu Ronbunshi/Journal Ceram. Soc. Japan. (1995). https://doi.org/10.2109/jcersj.103.315.

[59] U. Kumar, S. Upadhyay, Studies on dielectric and electrical properties of RuddlesdenPopper oxide Sr 2 SnO 4, Mater. Lett. 227 (2018) 100-103. https://doi.org/10.1016/j.matlet.2018.05.046. 